\title{
Elastic wave-mode separation for VTI media $a^{a}$
}

${ }^{a}$ Published in Geophysics, 74 , no. 5, WB19-WB32, (2009)

Jia Yan and Paul Sava

Center for Wave Phenomena

Colorado School of Mines 1

\begin{abstract}
Elastic wave propagation in anisotropic media is well represented by elastic wave equations. Modeling based on elastic wave equations characterizes both kinematics and dynamics correctly. However, because $\mathrm{P}$ and $\mathrm{S}$ modes are both propagated using elastic wave equations, there is a need to separate $\mathrm{P}$ and $\mathrm{S}$ modes to obtain clean elastic images. The separation of wave modes to $\mathrm{P}$ and $\mathrm{S}$ from isotropic elastic wavefields is typically done using Helmholtz decomposition. However, Helmholtz decomposition using conventional divergence and curl operators in anisotropic media does not give satisfactory results and leaves the different wave modes only partially separated. The separation of anisotropic wavefields requires the use of more sophisticated operators which depend on local material parameters. Anisotropic wavefield separation operators are constructed using the polarization vectors evaluated by solving the Christoffel equation at each point of the medium. These polarization vectors can be represented in the space domain as localized filtering operators, which resemble conventional derivative operators. The spatially-variable "pseudo" derivative operators perform well in heterogeneous VTI media even at places of rapid velocity/density variation. Synthetic results indicate that the operators can be used to separate wavefields for VTI media with an arbitrary degree of anisotropy.
\end{abstract}

\section{INTRODUCTION}

Wave equation migration for elastic data usually consists of two steps. The first step is wavefield reconstruction in the subsurface from data recorded at the surface. The second step is the application of an imaging condition which extracts reflectivity information from the reconstructed wavefields.

The elastic wave equation migration for multicomponent data can be implemented in two ways. The first approach is to separate recorded elastic data into compressional and transverse ( $\mathrm{P}$ and $\mathrm{S}$ ) modes and use the separated data for acoustic wave equation migration separately. This acoustic imaging approach to elastic waves is

\footnotetext{
${ }^{1}$ e-mail: jyan@mines.edu, psava@mines.edu
} 
more frequently used, but it is fundamentally based on the assumption that $\mathrm{P}$ and $\mathrm{S}$ data can be successfully separated on the surface, which is not always true (Etgen, 1988; Zhe and Greenhalgh, 1997). The second approach is to not separate P and S modes on the surface, but to extrapolate the entire elastic wavefield at once, and then separate wave modes prior to applying an imaging condition. The reconstruction of elastic wavefields can be implemented using various techniques, including reconstruction by time reversal (RTM) (Chang and McMechan, 1986, 1994) or by Kirchhoff integral techniques (Hokstad, 2000).

The imaging condition applied to the reconstructed vector wavefields directly determines the quality of the images. Conventional crosscorrelation imaging condition does not separate the wave modes and crosscorrelates the Cartesian components of the elastic. In general, the various wave modes (P and $\mathrm{S}$ ) are mixed on all wavefield components and cause crosstalk and image artifacts. Yan and Sava (2009) suggest using imaging conditions based on elastic potentials, which require crosscorrelation of separated modes. Potential-based imaging condition creates images that have clear physical meaning, in contrast with images obtained with Cartesian wavefield components, thus justifying the need for wave mode separation.

As the need for anisotropic imaging increases, more processing and migration are performed based on anisotropic acoustic one-way wave equations (Alkhalifah, 1998, 2000; Shan, 2006; Shan and Biondi, 2005; Fletcher et al., 2009; Fowler et al., 2010). However, much less research has been done on anisotropic elastic migration based on two-way wave equations. Elastic Kirchhoff migration (Hokstad, 2000) obtains pure-mode and converted mode images by downward continuation of elastic vector wavefields with a visco-elastic wave equation. The wavefield separation is effectively done with elastic Kirchhoff integration, which handles both P and S waves. However, Kirchhoff migration does not perform well in areas of complex geology where ray theory breaks down (Gray et al., 2001), thus requiring migration with more accurate methods, such as reverse time migration.

One of the complexities that impedes elastic wave equation anisotropic migration is the difficulty to separate anisotropic wavefields into different wave modes after reconstructing the elastic wavefields. However, the proper separation of anisotropic wave modes is as important for anisotropic elastic migration as is the separation of isotropic wave modes for isotropic elastic migration. The main difference between anisotropic and isotropic wavefield separation is that Helmholtz decomposition is only suitable for the separation of isotropic wavefields and is inadequate for anisotropic wavefields.

In this chapter, I show how to construct wavefield separators for VTI (vertical transverse isotropy) media applicable to models with spatially varying parameters. I apply these operators to anisotropic elastic wavefields and show that they successfully separate anisotropic wave modes, even for extremely anisotropic media.

The main application of this technique is in the development of elastic reverse time migration. In this case, complete wavefields containing both $\mathrm{P}$ and $\mathrm{S}$ wave modes are 
reconstructed from recorded data. The reconstructed wavefields are separated in pure wave modes prior to the application of a conventional crosscorrelation imaging condition. I limit the scope of this chapter only to the wave-mode separation procedure in highly heterogeneous media, although the ultimate goal of this procedure is to aid elastic RTM.

\section{SEPARATION METHOD}

Separation of scalar and vector potentials can be achieved by Helmholtz decomposition, which is applicable to any vector field $\mathbf{W}(x, y, z)$. By definition, the vector wavefield $\mathbf{W}$ can be decomposed into a curl-free scalar potential $\Theta$ and a divergencefree vector potential $\boldsymbol{\Psi}$ according to the relation (Aki and Richards, 2002):

$$
\mathbf{W}=\nabla \Theta+\nabla \times \mathbf{\Psi}
$$

Equation 1 is not used directly in practice, but the scalar and vector components are obtained indirectly by the application of the $\nabla \cdot$ and $\nabla \times$ operators to the extrapolated elastic wavefield:

$$
\begin{aligned}
P & =\nabla \cdot \mathbf{W}, \\
\mathbf{S} & =\nabla \times \mathbf{W} .
\end{aligned}
$$

For isotropic elastic fields far from the source, quantities $P$ and $\mathbf{S}$ describe compressional and shear wave modes, respectively (Aki and Richards, 2002).

Equations 2 and 3 allow one to understand why $\nabla \cdot$ and $\nabla \times$ pass compressional and transverse wave modes, respectively. In the discretized space domain, one can write:

$$
P=\nabla \cdot \mathbf{W}=D_{x}\left[W_{x}\right]+D_{y}\left[W_{y}\right]+D_{z}\left[W_{z}\right],
$$

where $D_{x}, D_{y}$, and $D_{z}$ represent spatial derivatives in the $x, y$, and $z$ directions, respectively. Applying derivatives in the space domain is equivalent to applying finite difference filtering to the functions. Here, $D[\cdot]$ represents spatial filtering of the wavefield with finite difference operators. In the Fourier domain, one can represent the operators $D_{x}, D_{y}$, and $D_{z}$ by $i k_{x}, i k_{y}$, and $i k_{z}$, respectively; therefore, one can write an equivalent expression to equation 4 as:

$$
\widetilde{\mathbf{P}}=i \mathbf{k} \cdot \widetilde{\mathbf{W}}=i k_{x} \widetilde{W}_{x}+i k_{y} \widetilde{W}_{y}+i k_{z} \widetilde{W}_{z}
$$

where $\mathbf{k}=\left\{k_{x}, k_{y}, k_{z}\right\}$ represents the wave vector, and $\widetilde{\mathbf{W}}\left(k_{x}, k_{y}, k_{z}\right)$ is the $3 \mathrm{D}$ Fourier transform of the wavefield $\mathbf{W}(x, y, z)$. We see that in this domain, the operator $i \mathbf{k}$ essentially projects the wavefield $\widetilde{\mathbf{W}}$ onto the wave vector $\mathbf{k}$, which represents the polarization direction for $\mathrm{P}$ waves. Similarly, the operator $\nabla \times$ projects the wavefield onto the direction orthogonal to the wave vector $\mathbf{k}$, which represents the polarization direction for $\mathrm{S}$ waves (Dellinger and Etgen, 1990). For illustration, Figure 1a shows the polarization vectors of the $\mathrm{P}$ mode of a $2 \mathrm{D}$ isotropic model as a function of normalized 

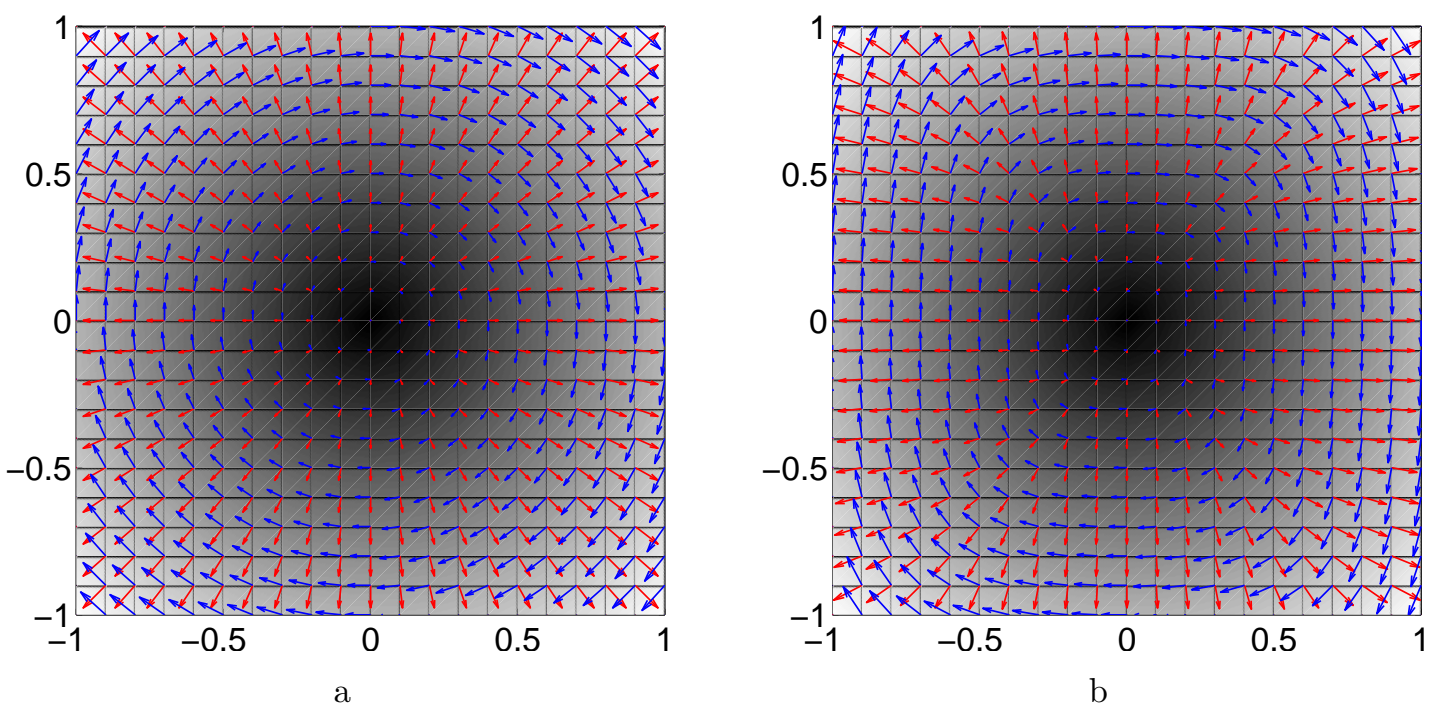

Figure 1: The $q \mathrm{P}$ and $q \mathrm{~S}$ polarization vectors as a function of normalized wavenumbers $k_{x}$ and $k_{z}$ ranging from -1 to +1 cycles, for (a) an isotropic model with $V_{P}=3 \mathrm{~km} / \mathrm{s}$ and $V_{S}=1.5 \mathrm{~km} / \mathrm{s}$, and (b) an anisotropic (VTI) model with $V_{P 0}=3 \mathrm{~km} / \mathrm{s}, V_{S 0}=$ $1.5 \mathrm{~km} / \mathrm{s}, \epsilon=0.25$ and $\delta=-0.29$. The red arrows are the $q \mathrm{P}$ wave polarization vectors, and the blue arrows are the $q \mathrm{~S}$ wave polarization vectors.

$k_{x}$ and $k_{z}$ ranging from -1 to 1 cycles. The polarization vectors are radial because the $\mathrm{P}$ waves in an isotropic medium are polarized in the same directions as the wave vectors.

Dellinger and Etgen (1990) suggest the idea that wave mode separation can be extended to anisotropic media by projecting the wavefields onto the directions in which the $\mathrm{P}$ and $\mathrm{S}$ modes are polarized. This requires that one should modify the wave separation equation 5 by projecting the wavefields onto the true polarization directions $\mathbf{U}$ to obtain quasi-P $(q \mathrm{P})$ waves:

$$
\widetilde{q P}=i \mathbf{U}(\mathbf{k}) \cdot \widetilde{\mathbf{W}}=i U_{x} \widetilde{W}_{x}+i U_{y} \widetilde{W}_{y}+i U_{z} \widetilde{W}_{z}
$$

In anisotropic media, $\mathbf{U}\left(k_{x}, k_{y}, k_{z}\right)$ is different from $\mathbf{k}$, as illustrated in Figure $1 \mathrm{~b}$, which shows the polarization vectors of $q \mathrm{P}$ wave mode for a $2 \mathrm{D}$ VTI anisotropic model with normalized $k_{x}$ and $k_{z}$ ranging from -1 to 1 cycles. Polarization vectors are not radial because $q \mathrm{P}$ waves in an anisotropic medium are not polarized in the same directions as wave vectors, except in the symmetry planes $\left(k_{z}=0\right)$ and along the symmetry axis $\left(k_{x}=0\right)$.

Dellinger and Etgen (1990) demonstrate wave mode separation in the wavenumber domain using projection of the polarization vectors, as indicated in equation 6 . However, for heterogeneous media, this equation is defective because the polarization vectors are spatially varying. One can write an equivalent expression to equation 6 
in the space domain for each grid point as:

$$
q P=\nabla_{a} \cdot \mathbf{W}=L_{x}\left[W_{x}\right]+L_{y}\left[W_{y}\right]+L_{z}\left[W_{z}\right],
$$

where $L_{x}, L_{y}$, and $L_{z}$ represent the inverse Fourier transforms of $i U_{x}, i U_{y}$, and $i U_{z}$, respectively. $L[\cdot]$ represents spatial filtering of the wavefield with anisotropic separators. $L_{x}, L_{y}$, and $L_{z}$ define the pseudo derivative operators in the $x, y$, and $z$ directions for an anisotropic medium, respectively, and they change from location to location according to the material parameters.

We obtain the polarization vectors $\mathbf{U}(\mathbf{k})$ by solving the Christoffel equation Aki and Richards, 2002; Tsvankin, 2005):

$$
\left[\mathbf{G}-\rho V^{2} \mathbf{I}\right] \mathbf{U}=0,
$$

where $\mathbf{G}$ is the Christoffel matrix $G_{i j}=c_{i j k l} n_{j} n_{l}$, in which $c_{i j k l}$ is the stiffness tensor, $n_{j}$ and $n_{l}$ are the normalized wave vector components in the $j$ and $l$ directions, $i, j, k, l=1,2,3$. The parameter $V$ corresponds to the eigenvalues of the matrix $\mathbf{G}$. The eigenvalues $V$ represent the phase velocities of different wave modes and are functions of the wave vector $\mathbf{k}$ (corresponding to $n_{j}$ and $n_{l}$ in the matrix $\mathbf{G}$ ). For plane waves propagating in any symmetry planes of a VTI medium, one can set $k_{y}$ to 0 and get

$$
\left[\begin{array}{ccc}
c_{11} k_{x}^{2}+c_{55} k_{z}^{2}-\rho V^{2} & 0 & \left(c_{13}+c_{55}\right) k_{x} k_{z} \\
0 & c_{66} k_{x}^{2}+c_{55} k_{z}^{2}-\rho V^{2} & 0 \\
\left(c_{13}+c_{55}\right) k_{x} k_{z} & 0 & c_{55} k_{x}^{2}+c_{33} k_{z}^{2}-\rho V^{2}
\end{array}\right]\left[\begin{array}{l}
U_{x} \\
U_{y} \\
U_{z}
\end{array}\right]=0
$$

The middle row of this matrix characterizes the $\mathrm{SH}$ wave polarized in the $y$ direction, and $q \mathrm{P}$ and $q \mathrm{SV}$ modes are uncoupled from the $\mathrm{SH}$ mode and are polarized in the vertical plane. The top and bottom rows of this equation allow one to compute the polarization vector $\mathbf{U}=\left\{U_{x}, U_{z}\right\}$ (the eigenvectors of the matrix ) of $\mathrm{P}$ or $\mathrm{SV}$ wave mode given the stiffness tensor at every location of the medium.

One can extend the procedure described here to heterogeneous media by computing two different operator for each mode at every grid point. In the symmetry planes of VTI media, the operators are 2D and depend on the local values of the stiffness coefficients. For each point, I pre-compute the polarization vectors as a function of the local medium parameters, and transform them to the space domain to obtain the wave mode separators. I assume that the medium parameters vary smoothly (locally homogeneous), but even for complex media, the localized operators work in the same way as the long finite difference operators. If one represents the stiffness coefficients using Thomsen parameters (Thomsen, 1986), then the pseudo derivative operators $L_{x}$ and $L_{z}$ depend on $\epsilon, \delta, V_{P 0}$ and $V_{S 0}$, which can be spatially varying parameters. One can compute and store the operators for all grid points in the medium, and then use these operators to separate $\mathrm{P}$ and $\mathrm{S}$ modes from reconstructed elastic wavefields at different time steps. Thus, wavefield separation in VTI media can be achieved simply by non-stationary filtering with operators $L_{x}$ and $L_{z}$. 


\section{OPERATOR PROPERTIES}

In this section, I discuss the properties of the anisotropic "derivative" operators, including order of accuracy, size, and compactness.

\section{Operator orders}

As I have showed in the previous section, the isotropic separation operators (divergence and curl) in equations 4 and 5 are exact in the $x$ and $k$ domains. The exact derivative operators are infinitely long series in the discretized space domain. In practice, when evaluating the derivatives numerically, one needs to take some approximations to make the operators short and computationally efficient. Usually, difference operators are evaluated at different orders of accuracy. The higher order the approximation is, the more accurate and longer the operator becomes. For example, the $2^{\text {nd }}$ order operator has coefficients $\left(-\frac{1}{2},+\frac{1}{2}\right)$, and the more accurate $4^{\text {th }}$ order operator has coefficients $\left(+\frac{1}{12},-\frac{2}{3}, \frac{2}{3},-\frac{1}{12}\right)$ (Fornberg and Ghrist, 1999).

In the wavenumber domain, for isotropic media, as shown by the black line in Figure 2(b), the exact difference operator is $i k$. Appendix A shows the $k$ domain equivalents of the $2^{\text {nd }}, 4^{\text {th }}, 6^{\text {th }}$, and $8^{\text {th }}$ order finite difference operators, and they are plotted in Figure 2(b). The higher order operators have responses closer to the exact operator $i k$ (black line). To obtain vertical and horizontal derivatives of different orders of accuracy, I weight the polarization vector $i \mathbf{k}$ components $i k_{x}$ and $i k_{z}$ by the weights shown in Figure 2(c). For VTI media, similarly, I weight the anisotropic polarization vector $i \mathbf{U}(\mathbf{k})$ components $i U_{x}$ and $i U_{z}$ by these same weights. The weighted vectors are then transformed back to space domain to obtain the anisotropic stencils.

\section{Operator size and compactness}

Figure 3 shows the derivative operators of $2^{\text {nd }}, 4^{\text {th }}, 6^{\text {th }}$, and $8^{\text {th }}$ orders in the $z$ and $x$ directions for isotropic and VTI $(\epsilon=0.25, \delta=-0.29)$ media. As we can see, isotropic operators become longer when the order of accuracy is higher. Anisotropic operators, however, do not change much in size. One can see that the central parts of the anisotropic operators look similar to their corresponding isotropic operators and change with the order of accuracy; while the outer parts of these anisotropic operators all look similar, and do not change much with the order of accuracy. This indicates that the central parts of the operators are determined by the order of accuracy, while the outer parts are representation of the degree of anisotropy.

Figure 4 shows anisotropic derivative operators with same order of accuracy $\left(8^{\text {th }}\right.$ order in space) for three VTI media with different combinations of $\epsilon$ and $\delta$. These operators have similar central parts, but different outer parts. This result is consistent with the previous observation that the central part of an operator is determined by the order of accuracy, and the outer part is controlled by anisotropy parameters. 

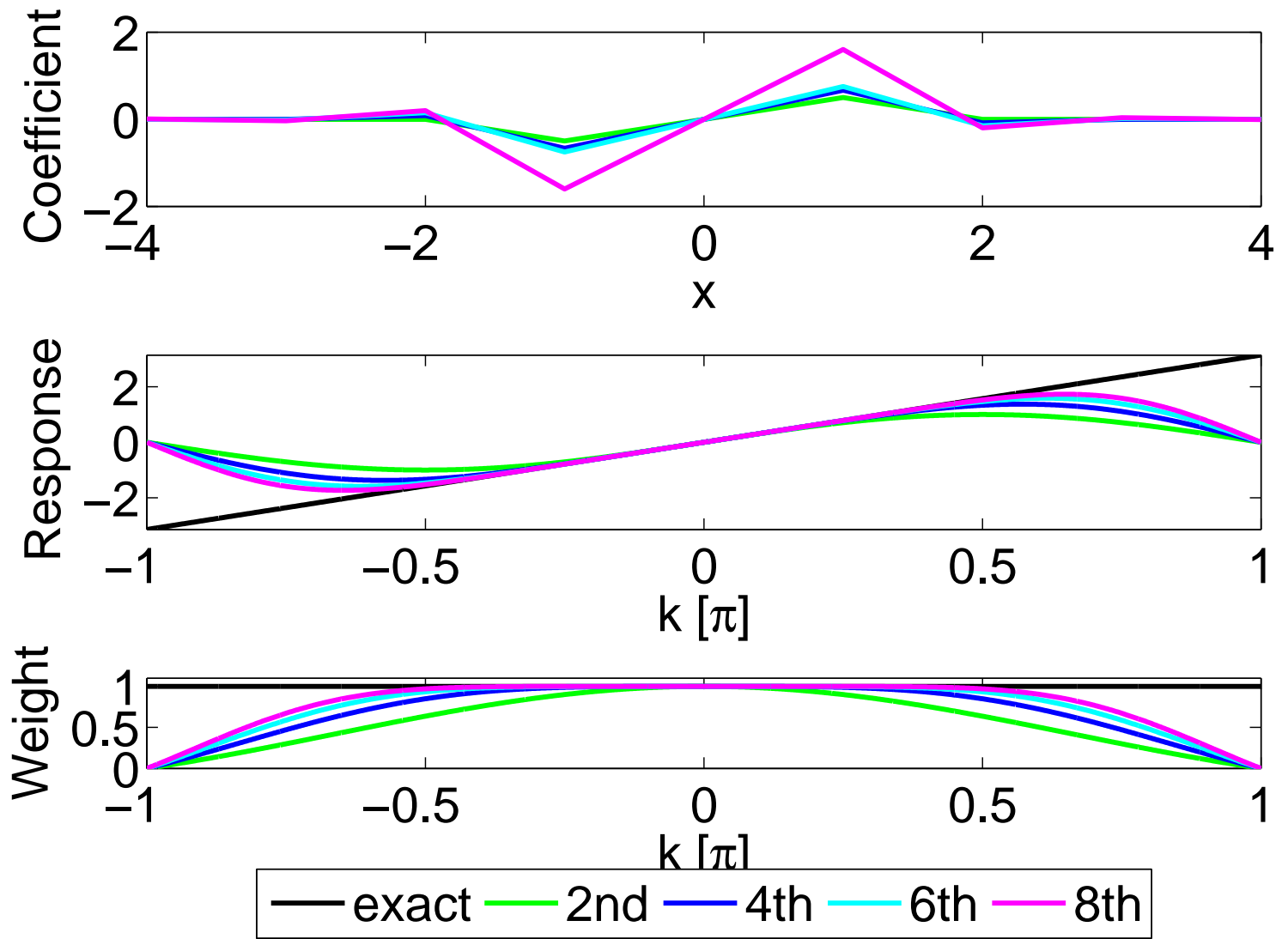

Figure 2: Comparison of derivative operators of different orders of accuracy $\left(2^{\text {nd }}\right.$, $4^{\text {th }}, 6^{\text {th }}$, and $8^{\text {th }}$ orders in space, as well as the approximation applied in Dellinger and Etgen (1990)-cosine taper) in both (a) the $x$ domain and (b) the $k$ domain. (c) Weights to apply to the components of the polarization vectors. 

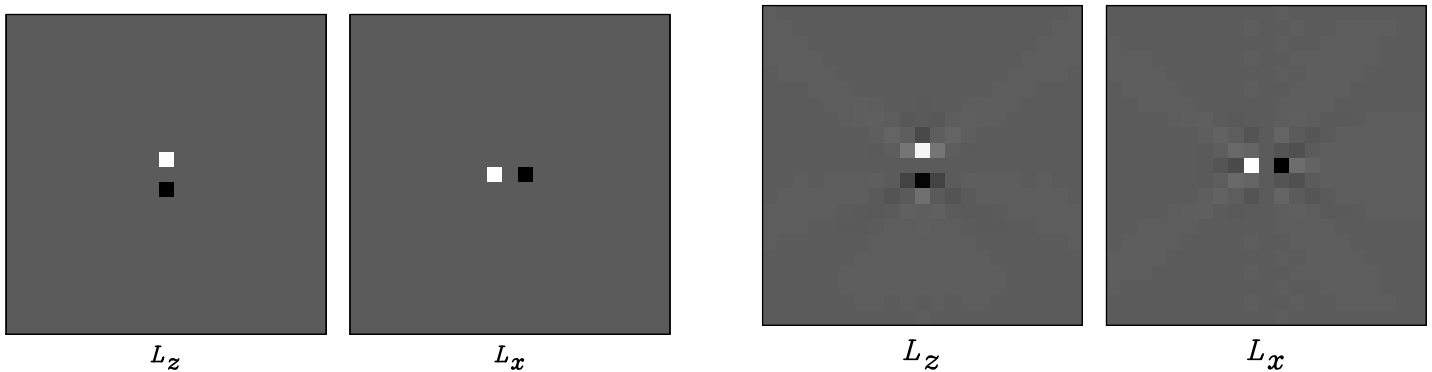

a

$\mathrm{b}$
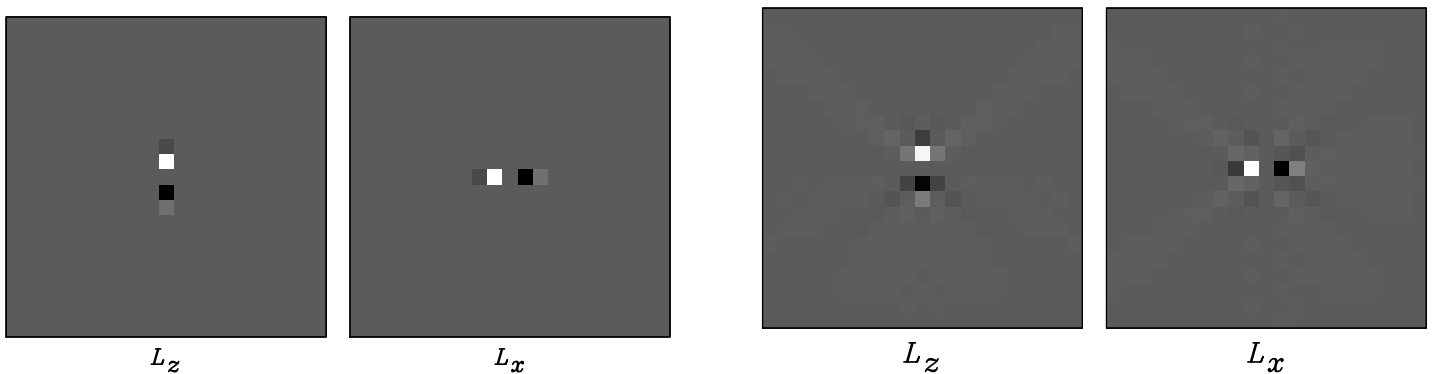

d
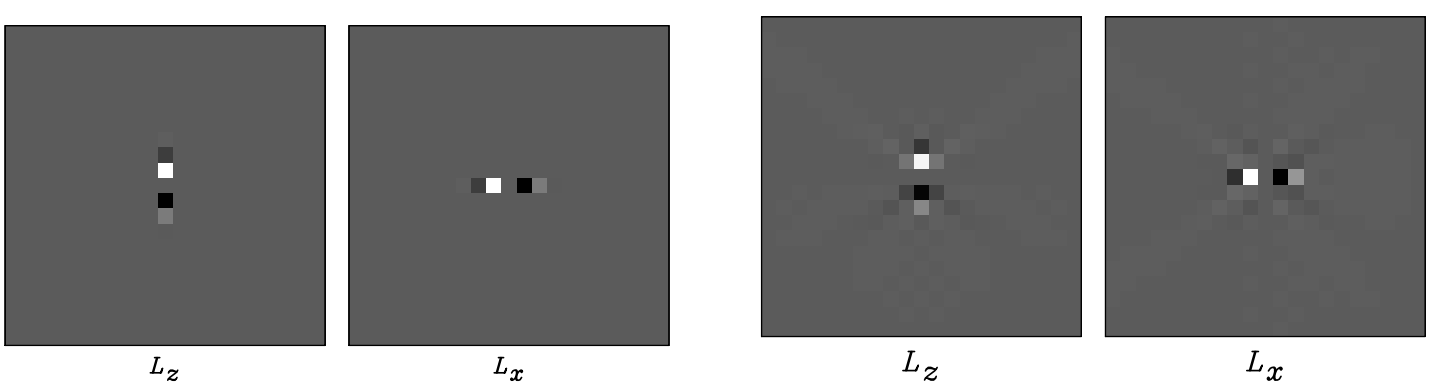

f
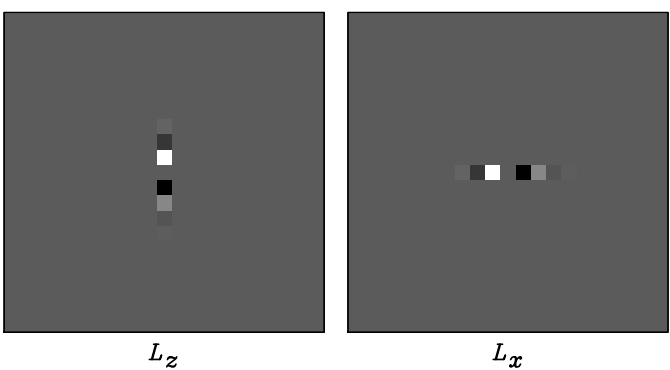

g

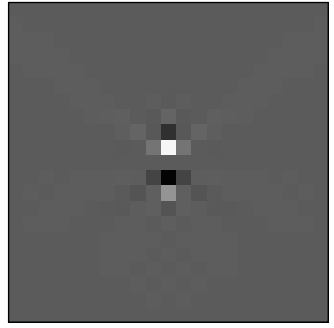

$L_{z}$

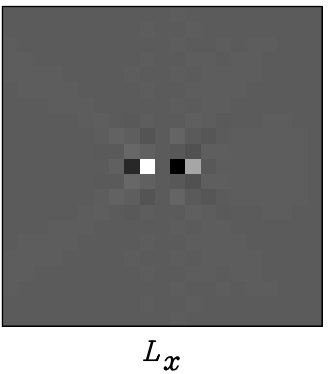

$\mathrm{h}$

Figure 3: $2^{\text {nd }}, 4^{\text {th }}, 6^{\text {th }}$, and $8^{\text {th }}$ order derivative operators for an isotropic medium $\left(V_{P}=3 \mathrm{~km} / \mathrm{s}\right.$ and $\left.V_{S}=1.5 \mathrm{~km} / \mathrm{s}\right)$ and a VTI medium $\left(V_{P 0}=3 \mathrm{~km} / \mathrm{s}, V_{S 0}=1.5 \mathrm{~km} / \mathrm{s}\right.$, $\epsilon=0.25$ and $\delta=-0.29)$. The left column includes isotropic operators, and the right column includes anisotropic operators. From top to bottom are operators with increasing orders of accuracy. 
Figure $5 \mathrm{a}$ shows the influence of approximation to finite difference $\left(2^{\text {nd }}\right.$ and $8^{\text {th }}$ order, Figures 3h and b). The "anisotropic" part ("diagonal tails") are almost the same, and the difference comes from the central part. Figure $5 b$ shows the difference between operators with different anisotropy (Figures $4 \mathrm{a}$ and b). The difference mainly lies in the "tails" of the operators.

A comparison between Figures $4 \mathrm{a}$ and $\mathrm{b}$ shows that when one has large difference between $\epsilon$ and $\delta$, the operator is big in size and when the difference of $\epsilon$ and $\delta$ stays the same, the parameter $\delta$ affects the operator size. A comparison between Figures $4 \mathrm{~b}$ and $\mathrm{C}$ shows that when the difference between $\epsilon$ and $\delta$ becomes smaller and $\delta$ does not change, the operator get smaller in size. This result is consistent with the polarization equation for VTI media with weak anisotropy (Tsvankin, 2005):

$$
\nu_{P}=\theta+B\left[\delta+2(\epsilon-\delta) \sin ^{2} \theta\right] \sin 2 \theta,
$$

where

$$
B \equiv \frac{1}{2 f}=\frac{1}{2\left(1-V_{S 0}^{2} / V_{P 0}^{2}\right)} .
$$

$V_{P 0}$ and $V_{S 0}$ are vertical $\mathrm{P}$ and $\mathrm{S}$ wave velocities, $\theta$ is the phase angle, and $\nu_{p}$ is the $\mathrm{P}$ wave polarization angle. This equation demonstrates the deviation of anisotropic polarization vectors with the isotropic ones: difference of $\epsilon$ and $\delta$ (which is approximately $\eta$ for weak anisotropy) and the parameter $\delta$ control the deviation of $\nu_{P}$ from $\theta$ and therefore the size of the anisotropic derivative operators.

\section{Operator truncation}

The derivative operators for isotropic and anisotropic media are very different in both shape and size, and the operators vary with the strength of anisotropy. In theory, analytic isotropic derivatives are point operators in the continuous limit. If one can do perfect Fourier transform to $i k_{x}$ and $i k_{z}$ (without doing the approximations to different orders of accuracy as one does in Figure 2), one gets point derivative operators. This is because $i k_{x}$ is constant in the $z$ direction (see Figure 6a), whose Fourier transform is delta function; the exact expression of $i k_{x}$ in the $k$ domain also makes the operator point in the $x$ direction. This makes the isotropic derivative operators point operators in the $x$ and $z$ direction. And when one applies approximations to the operators, they are compact in the space domain.

However, even if one does perfect Fourier transformation to $i U_{x}$ and $i U_{z}$ (without doing the approximations for different orders of accuracy) for VTI media, the operators will not be point operators because $i U_{x}$ and $i U_{z}$ are not constants in $z$ and $x$ directions, respectively (see Figure 6b). The $x$ domain operators spread out in all directions (Figures $3 \mathrm{~b}, \mathrm{~d}, \mathrm{f}$, and $\mathrm{h}$ ).

This effect is illustrated by Figure 3. When the order of accuracy decreases, the isotropic operators become more compact (shorter in space), while the anisotropic operators do not get more compact. No matter how one improves the compactness 

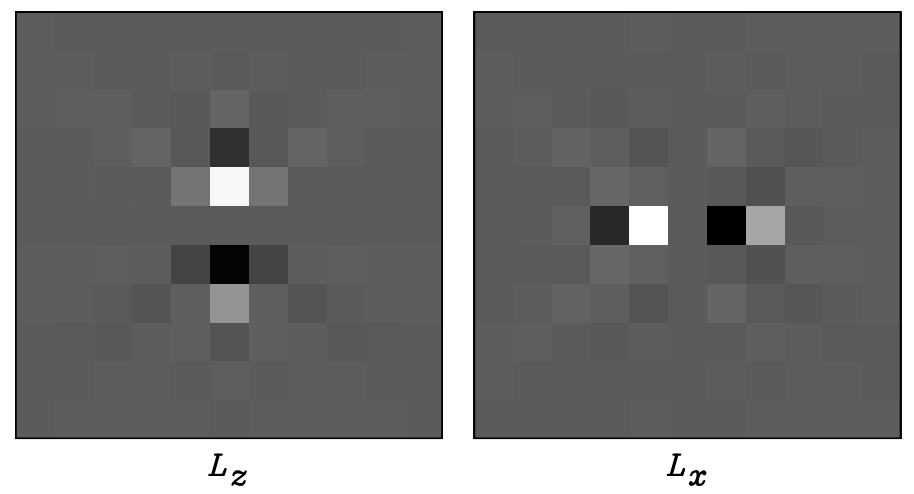

a
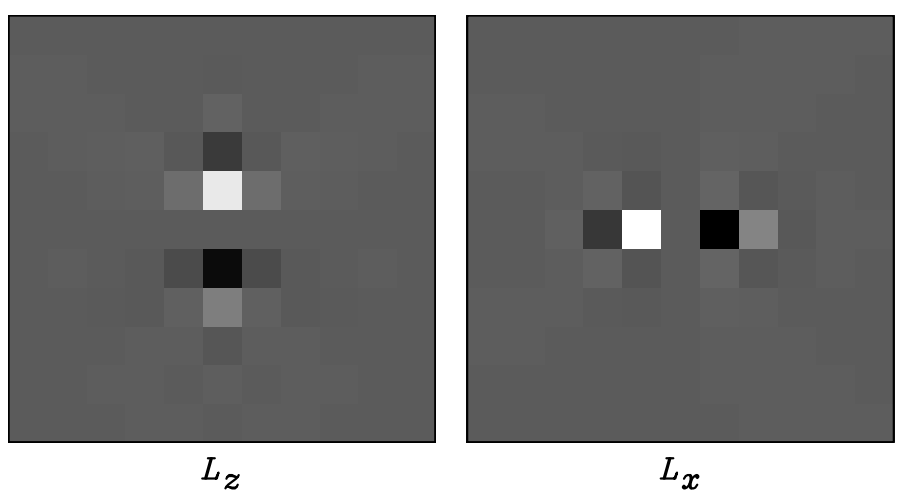

$\mathrm{b}$
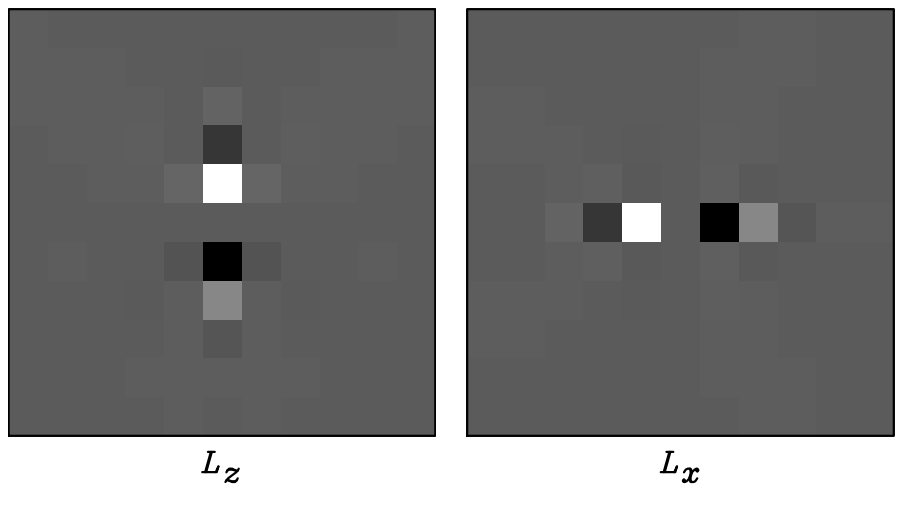

Figure 4: $8^{\text {th }}$ order anisotropic pseudo derivative operators for three VTI media: a) $\epsilon=0.25, \delta=-0.29$, b) $\epsilon=0.54, \delta=0$, and c) $\epsilon=0.2, \delta=0$. 

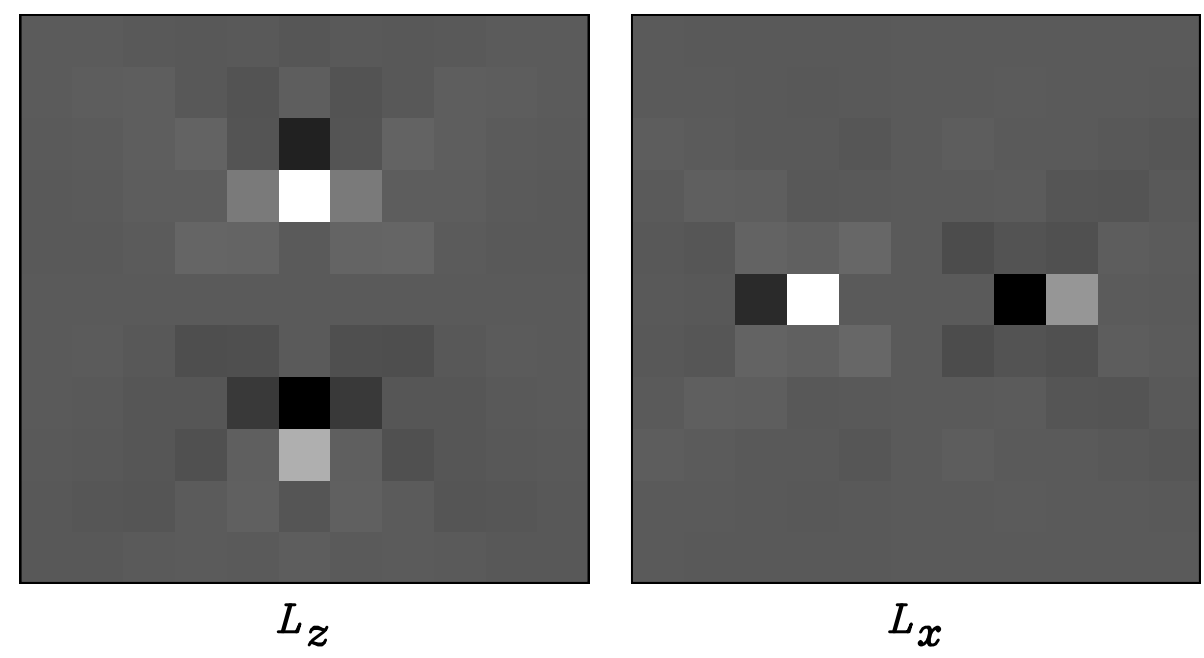

a
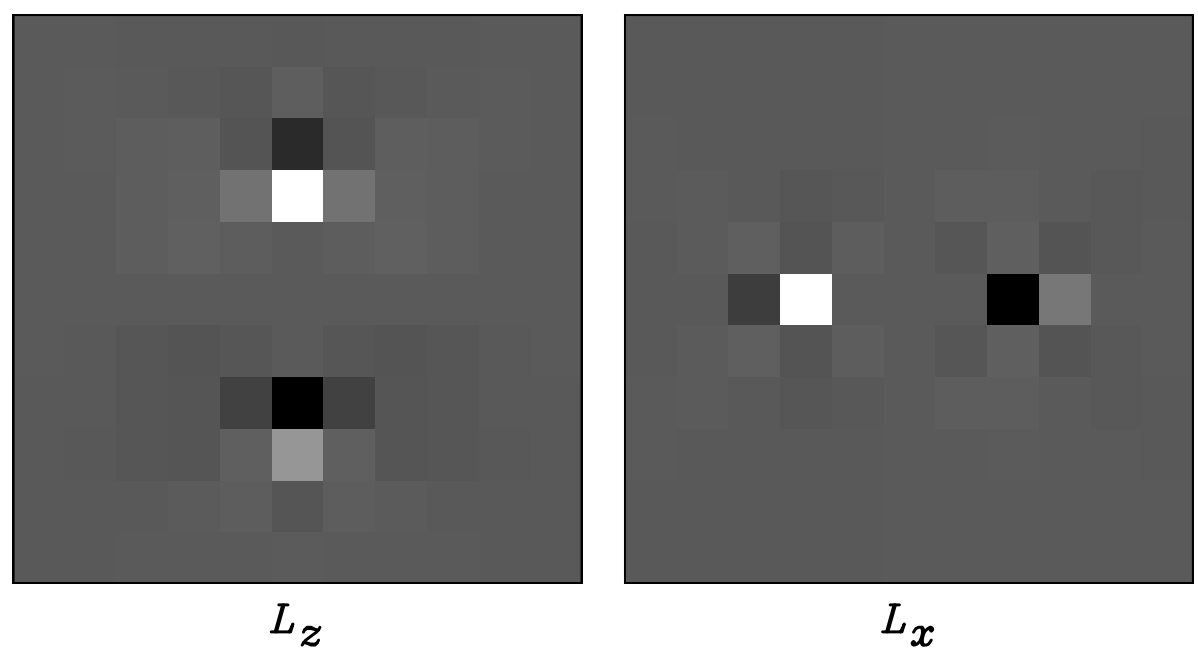

$\mathrm{b}$

Figure 5: (a) The difference between the $8^{\text {th }}$ and $2^{\text {nd }}$ order operators (Figures $3 \mathrm{~h}$ and b) for a VTI medium with anisotropy $\epsilon=0.25, \delta=-0.29$ in the $z$ and $x$ directions. (b) The difference between the $8^{\text {th }}$ order anisotropic operators for a VTI medium with anisotropy $\epsilon=0.25, \delta=-0.29$ (Figure 4a) and a VTI medium with anisotropy $\epsilon=0.54$, $\delta=0$ (Figure 4b). 


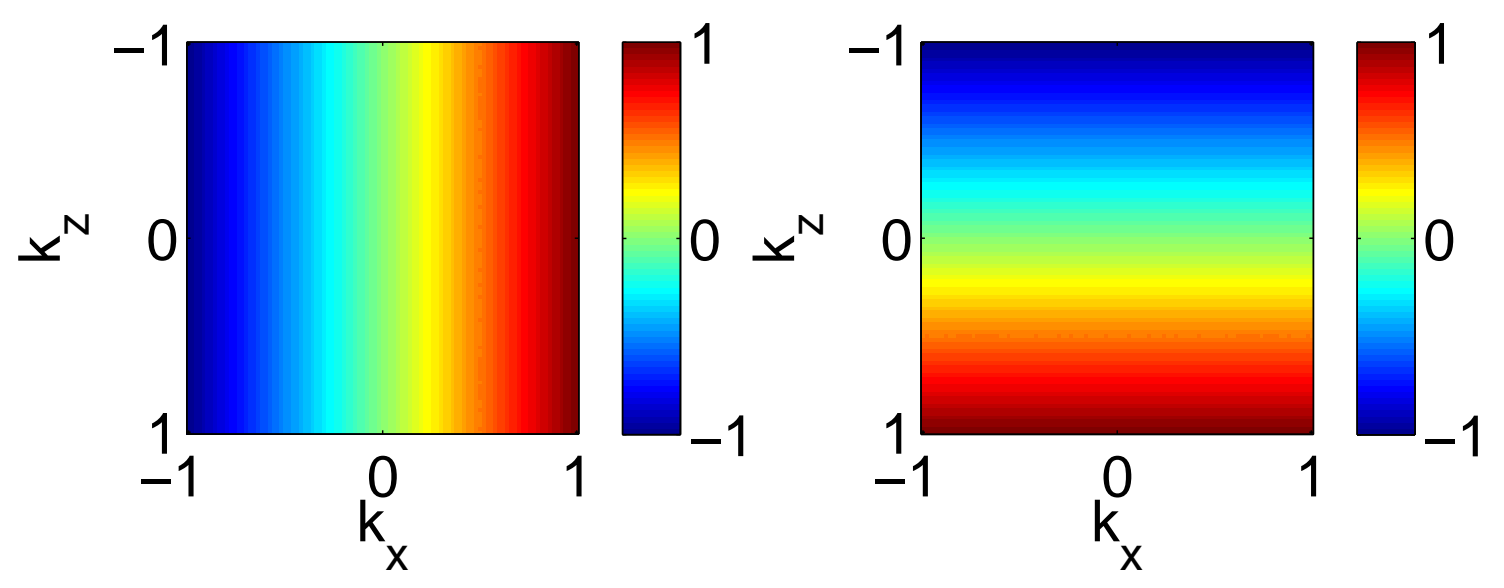

a
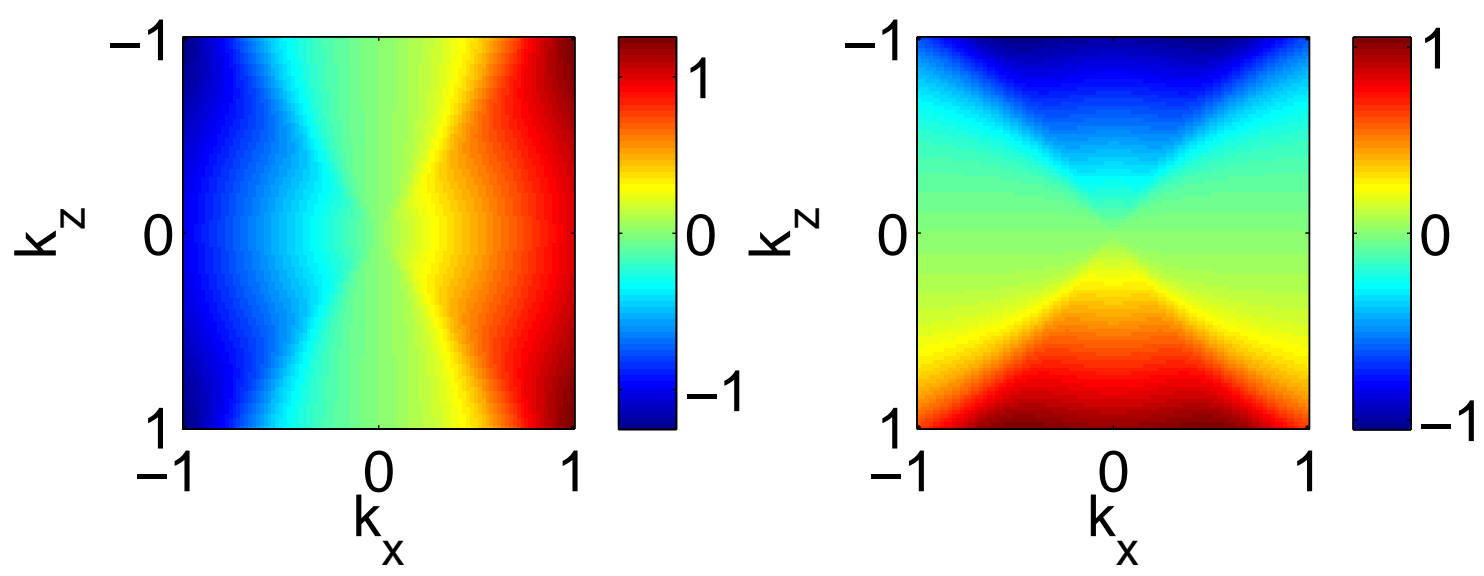

$\mathrm{b}$

Figure 6: (a) Isotropic and (b) VTI $(\epsilon=0.25, \delta=-0.29)$ polarization vectors (Figure 21 projected on to the $x$ (left column) and $z$ directions (right column). The isotropic polarization vectors components in the $z$ and $x$ directions depend only on $k_{z}$ and $k_{x}$, respectively. In contrast, the anisotropic polarization vectors components are functions of both $k_{x}$ and $k_{z}$. 
of isotropic operators, one does not get compact anisotropic operators in the space domain by the same means.

Because the size of the anisotropic derivative operators is usually large, it is natural that one would truncate the operators to save computation. Figure 7 shows a snapshot of an elastic wavefield and corresponding derivative operators for a VTI medium with $\epsilon=0.25$ and $\delta=-0.29$. Figure 8 shows the attempt of separation using truncated operator size of (a) $11 \times 11$, (b) $31 \times 31$ and (c) $51 \times 51$ out of the full operator size $65 \times$ 65 . Figure 8 shows that the truncation causes the wave-modes incompletely separated. This is because the truncation changes the directions of the polarization vectors, thus projecting the wavefield displacements onto wrong directions. Figure 9 presents the $\mathrm{P}$-wave polarization vectors before and after the truncation. For a truncated operator size of $11 \times 11$, the polarization vectors deviate from the correct ones to a maximum of $10^{\circ}$, but even this difference makes the separation incomplete.

\section{EXAMPLES}

I illustrate the anisotropic wave mode separation with a simple synthetic example and a more challenging elastic Sigsbee 2A model (Paffenholz et al., 2002).

\section{Simple model}

I consider a 2D isotropic model characterized by the $V_{P}, V_{S}$ and density shown in Figures $10 \mathrm{a}, \mathrm{c}$. The model contains negative $\mathrm{P}$ and $\mathrm{S}$ velocity anomalies that triplicate the wavefields. The source is located at the center of the model. Figure 11a shows the vertical and horizontal components of one snapshot of the simulated elastic wavefield (generated using the $8^{\text {th }}$ order finite difference solution of the elastic wave equation), Figure $11 \mathrm{~b}$ shows the separation to $\mathrm{P}$ and $\mathrm{S}$ modes using $\nabla \cdot$ and $\nabla \times$ operators, and Figure $11 \mathrm{c}$ shows the mode separation obtained using the pseudo operators which are dependent on the medium parameters. A comparison of Figures $11 \mathrm{~b}$ and $\mathrm{c}$ indicates that the $\nabla \cdot$ and $\nabla \times$ operators and the pseudo operators work identically well for this isotropic medium.

I then consider a 2D anisotropic model similar to the previous model shown in Figures $10 \mathrm{a}$ c (with $V_{P}, V_{S}$ representing the vertical $\mathrm{P}$ and $\mathrm{S}$ wave velocities), and additionally characterized by the parameters $\epsilon$ and $\delta$ shown in Figures 10d and e, respectively. The parameters $\epsilon$ and $\delta$ vary gradually from top to bottom and left to right, respectively. The upper left part of the medium is isotropic and the lower right part is highly anisotropic. Since the difference of $\epsilon$ and $\delta$ is great at the bottom part of the model, the $q \mathrm{~S}$ waves in this region are severely triplicated due to this strong anisotropy.

Figure 12 illustrates the pseudo derivative operators obtained at different locations in the model defined by the intersections of $x$ coordinates $0.3,0.6,0.9 \mathrm{~km}$ and $z$ 

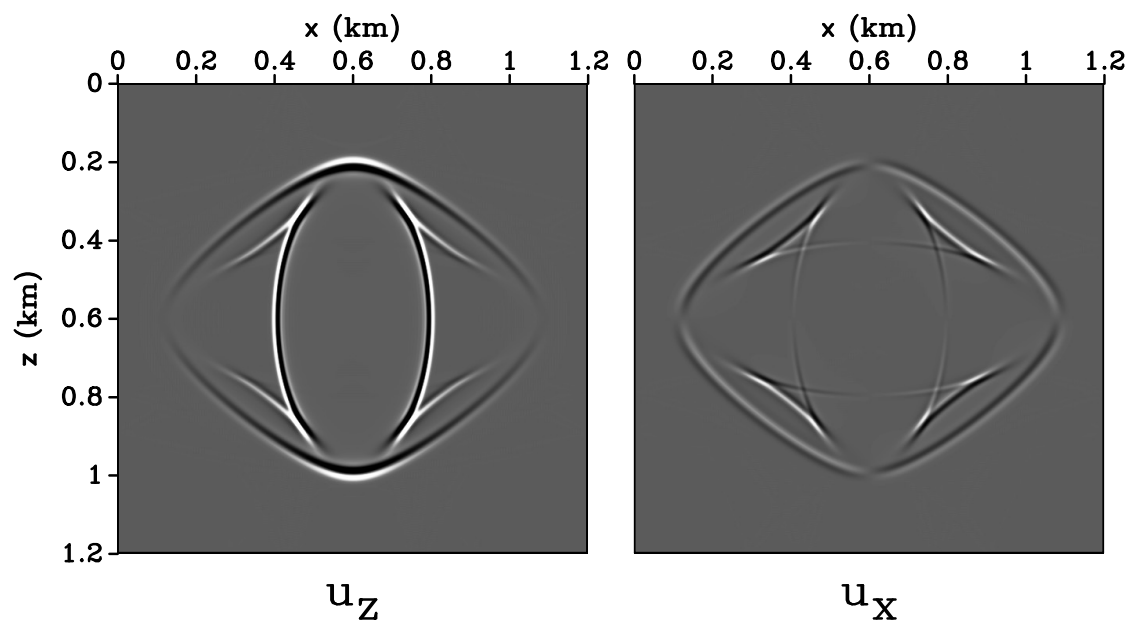

a

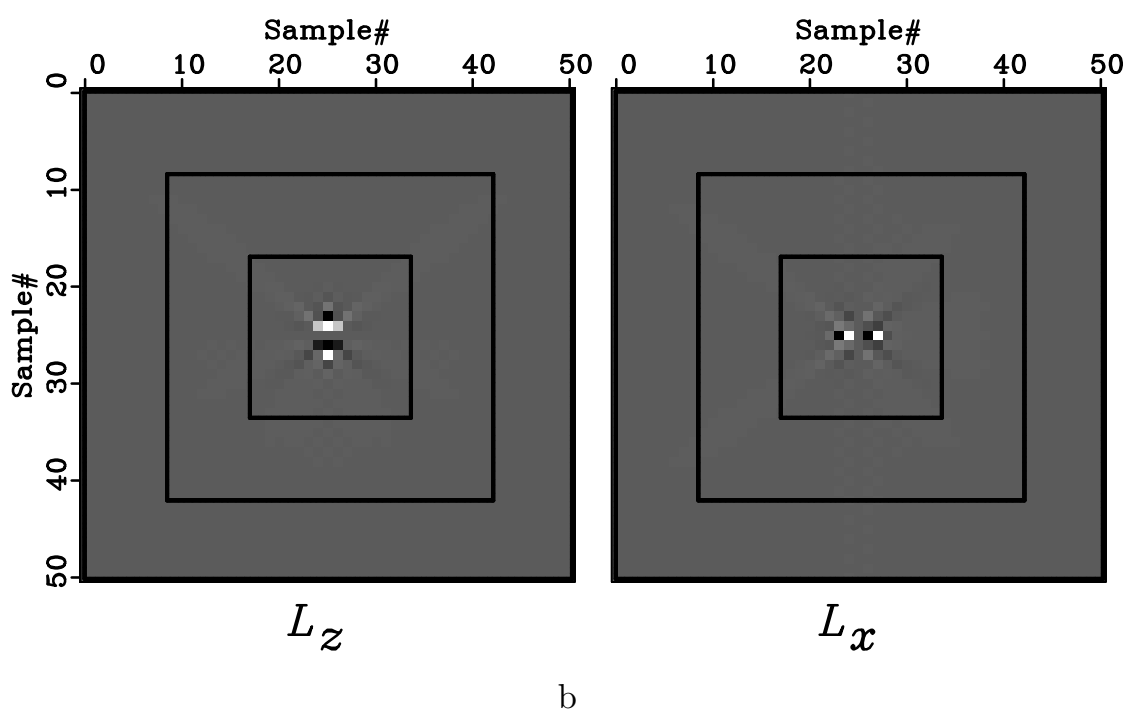

Figure 7: (a) A snapshot of an elastic wavefield showing the vertical (left) and horizontal (right) components for a VTI medium $(\epsilon=0.25$ and $\delta=-0.29)$. (b) $8^{\text {th }}$ order anisotropic pseudo derivative operators in $z$ (left) and $x$ (right) direction for this VTI medium. The boxes show the truncation of the operator to sizes of $11 \times 11,31 \times 31$, and $51 \times 51$. 


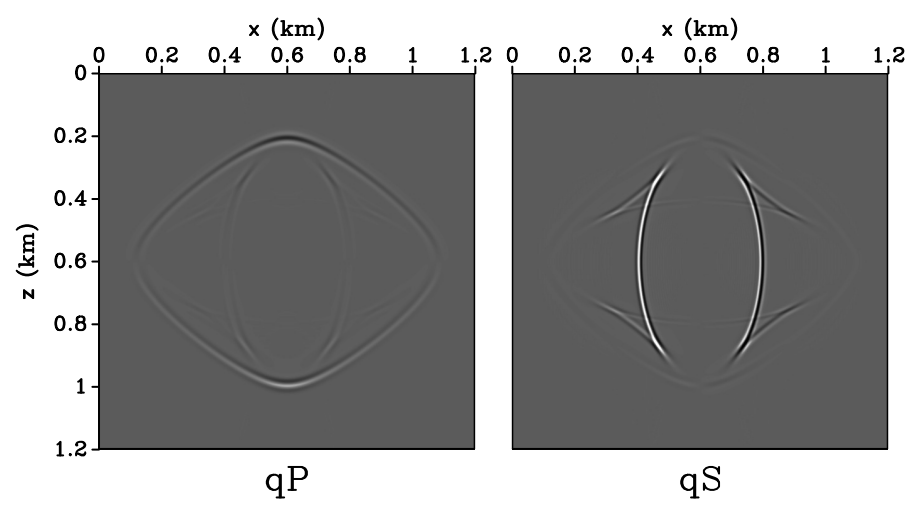

a
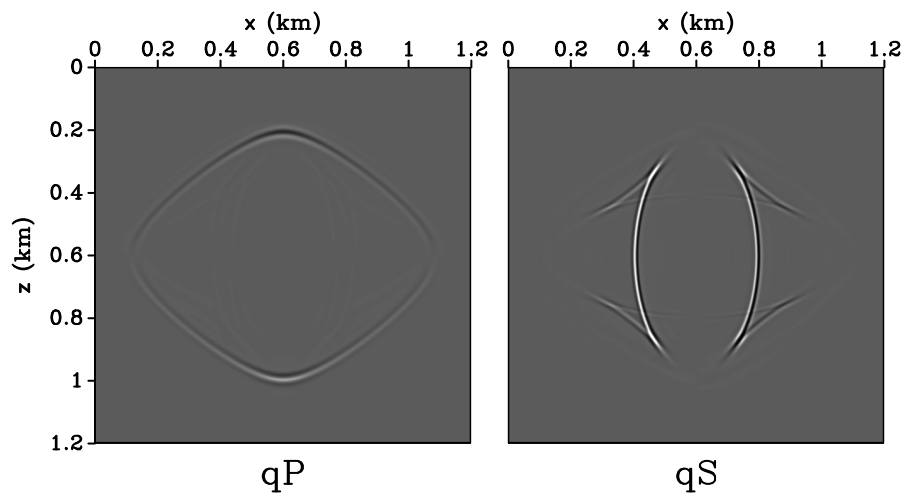

$\mathrm{b}$
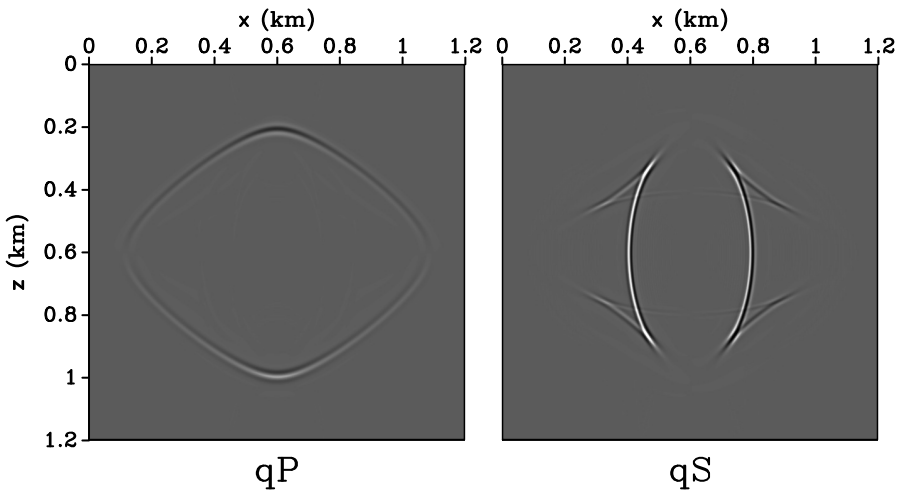

$\mathrm{C}$

Figure 8: separation by $8^{\text {th }}$ order anisotropic pseudo derivative operators of different sizes: (a) $11 \times 11$, (b) $31 \times 31$, (c) $51 \times 51$, shown in Figure $7 \mathrm{~b}$. The plot shows the larger the size of the operators, the better the separation is. 

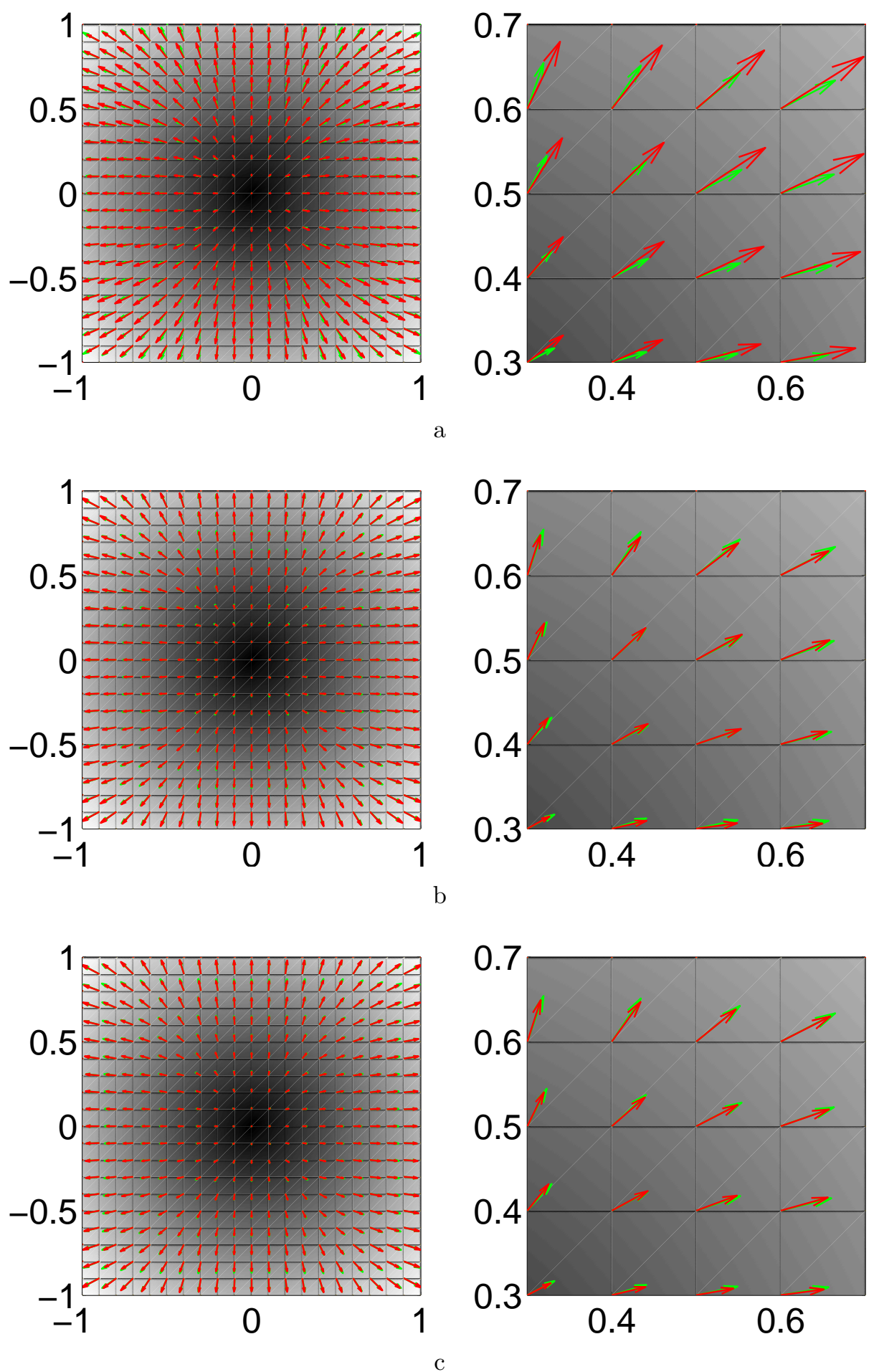

Figure 9: The deviation of polarization vectors by truncating the size of the spacedomain operator to (a) $11 \times 11$, (b) $31 \times 31$, (c) $51 \times 51$ out of $65 \times 65$. The left column shows polarization vectors from -1 to +1 cycles in both $x$ and $z$ directions, and the right column zooms to 0.3 to 0.7 cycles. The green vectors are the exact polarization vectors, and the red ones are the effective polarization vectors after truncation of the operator in the $x$ domain. 
coordinates $0.3,0.6,0.9 \mathrm{~km}$. Since the operators correspond to different combination of the parameters $\epsilon$ and $\delta$, they have different forms. The isotropic operator at coordinates $x=0.3 \mathrm{~km}$ and $z=0.3 \mathrm{~km}$, shown in Figure 12a, is purely vertical and horizontal, while the anisotropic operators (Figure $12 \mathrm{~b}$ to i) have "tails" radiating from the center. The operators become larger at locations where the medium is more anisotropic, for example, at coordinates $x=0.9 \mathrm{~km}$ and $z=0.9 \mathrm{~km}$.

Figure 13a shows the vertical and horizontal components of one snapshot of the simulated elastic anisotropic wavefield, Figure $13 \mathrm{~b}$ shows the separation to $q \mathrm{P}$ and $q \mathrm{~S}$ modes using conventional isotropic $\nabla \cdot$ and $\nabla \times$ operators, and Figure $13 \mathrm{c}$ shows the mode separation obtained using the pseudo operators constructed using the local medium parameters. A comparison of Figure $13 \mathrm{~b}$ and $13 \mathrm{c}$ indicates that the spatiallyvarying derivative operators successfully separate the elastic wavefields into $q \mathrm{P}$ and qS modes, while the $\nabla$. and $\nabla \times$ operators only work in the isotropic region of the model.

\section{Sigsbee model}

My second model (Figure 14) uses an elastic anisotropic version of the Sigsbee 2A model (Paffenholz et al., 2002). In the modified model, $V_{P 0}$ is taken from the original model, the $V_{P 0} / V_{S 0}$ ratio ranges from 1.5 to 2 , the parameter $\epsilon$ ranges from 0 to 0.48 (Figure $14 \mathrm{~d}$ ) and the parameter $\delta$ ranges 0 from to 0.10 (Figure 14e). The model is isotropic in the salt and the top part of the model. A vertical point force source is located at coordinates $x=14.5 \mathrm{~km}$ and $z=5.3 \mathrm{~km}$ to simulate the elastic anisotropic wavefield.

Figure 15 shows one snapshot of the modeled elastic anisotropic wavefields using the model shown in Figure 14. Figure 16 illustrates the separation of the anisotropic elastic wavefields using the $\nabla \cdot$ and $\nabla \times$ operators, and Figure 17 illustrates the separation using my pseudo derivative operators. Figure 16 shows the residual of unseparated $\mathrm{P}$ and $\mathrm{S}$ wave modes, such as at coordinates $x=13 \mathrm{~km}$ and $z=7 \mathrm{~km}$ in the $q \mathrm{P}$ panel and at $x=11 \mathrm{~km}$ and $z=7 \mathrm{~km}$ in the $q \mathrm{~S}$ panel. The residual of $\mathrm{S}$ waves in the $q \mathrm{P}$ panel of Figure 16 is very significant because of strong reflections from the salt bottom. This extensive residual can be harmful to under-salt elastic or even acoustic migration, if not removed completely. In contrast, Figure 17 shows the $q \mathrm{P}$ and $q \mathrm{~S}$ modes better separated, demonstrating the effectiveness of the anisotropic pseudo derivative operators constructed using the local medium parameters. These wavefields composed of well separated $q \mathrm{P}$ and $q \mathrm{~S}$ modes are essential to producing clean seismic images.

In order to test the separation with a homogeneous assumption of anisotropy in the model, I show in Figure 18 the separation with $\epsilon=0.3$ and $\delta=0.1$ in the $k$ domain. This separation assumes a model with homogeneous anisotropy. The separation shows that there is still residual in the separated panels. Although the residual is much weaker compared to separating using an isotropic model, it is still 


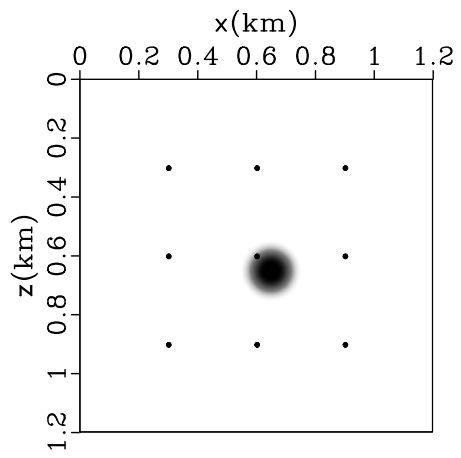

a

$\mathrm{x}(\mathrm{km})$

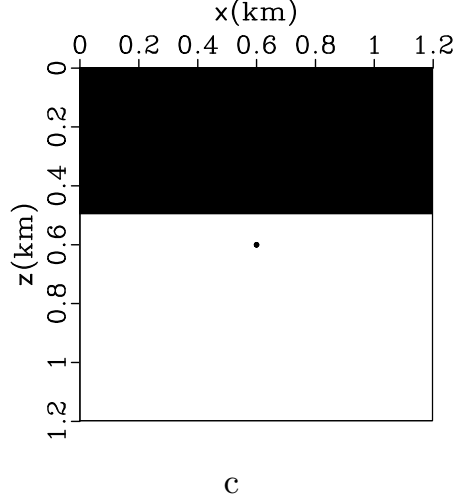

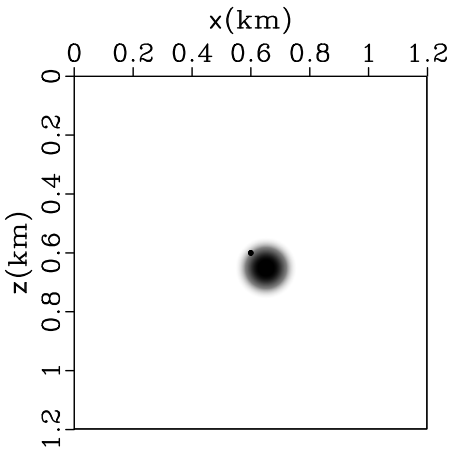

b

$\mathrm{x}(\mathrm{km})$

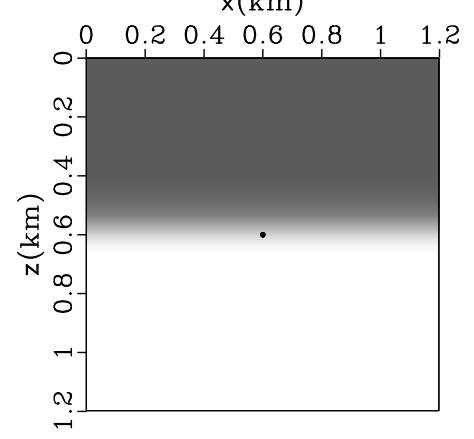

d

$\times(\mathrm{km})$

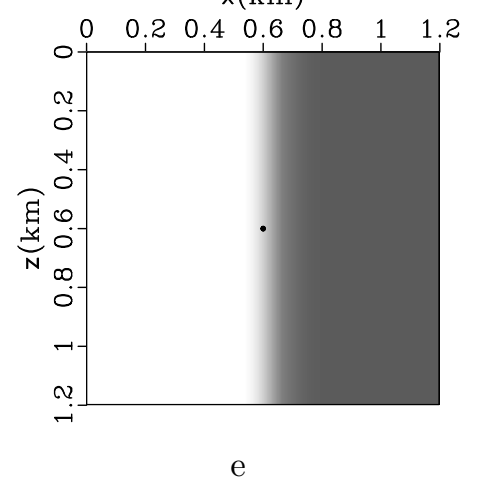

Figure 10: A $1.2 \mathrm{~km} \times 1.2 \mathrm{~km}$ model with parameters (a) $V_{p 0}=3 \mathrm{~km} / \mathrm{s}$ except for a low velocity Gaussian anomaly around $x=0.65 \mathrm{~km}$ and $z=0.65 \mathrm{~km}$, (b) $V_{S 0}=1.5 \mathrm{~km} / \mathrm{s} \mathrm{except} \mathrm{for} \mathrm{a} \mathrm{low} \mathrm{velocity} \mathrm{Gaussian} \mathrm{anomaly} \mathrm{around} x=0.65 \mathrm{~km}$ and $z=0.65 \mathrm{~km}$, (c) $\rho=1.0 \mathrm{~g} / \mathrm{cm}^{3}$ in the top layer and $2.0 \mathrm{~g} / \mathrm{cm}^{3}$ in the bottom layer, (d) $\epsilon$ smoothly varying from 0 to 0.25 from top to bottom, (e) $\delta$ smoothly varying from 0 to -0.29 from left to right. A vertical point force source is located at $x=0.6 \mathrm{~km}$ and $z=0.6 \mathrm{~km}$ shown by the dot in panels (b), (c), (d), and (e). The dots in panel (a) correspond to the locations of the anisotropic operators shown in Figure 12 . 

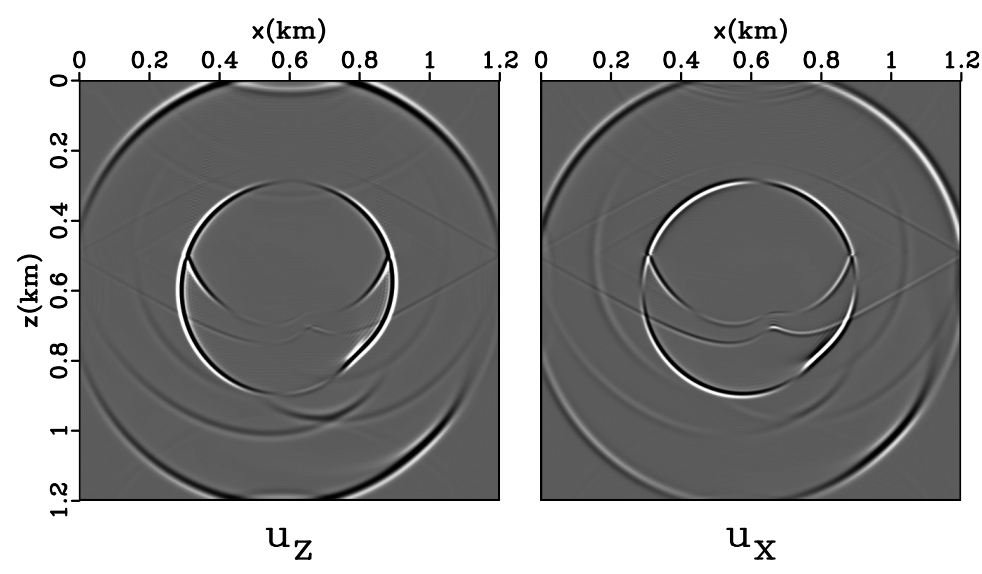

a
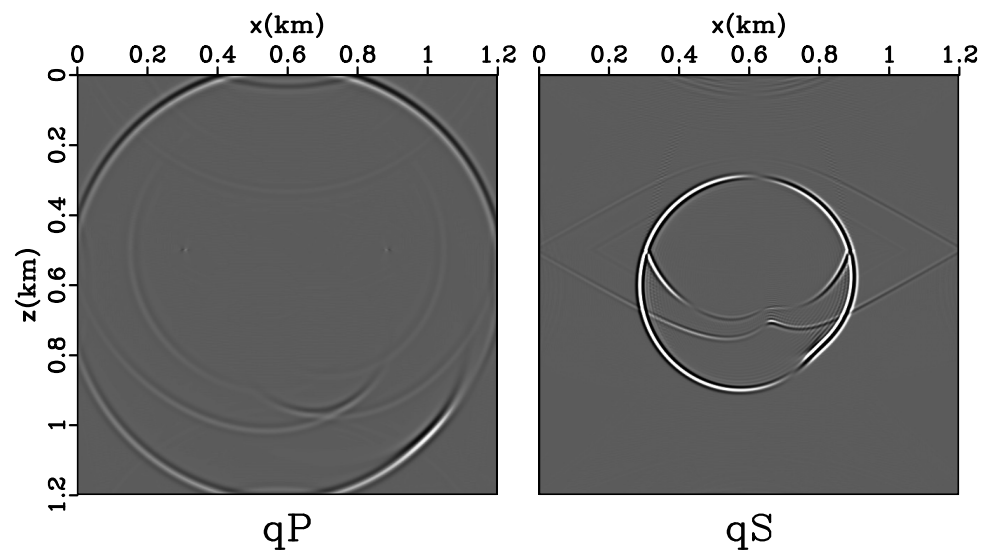

$\mathrm{b}$

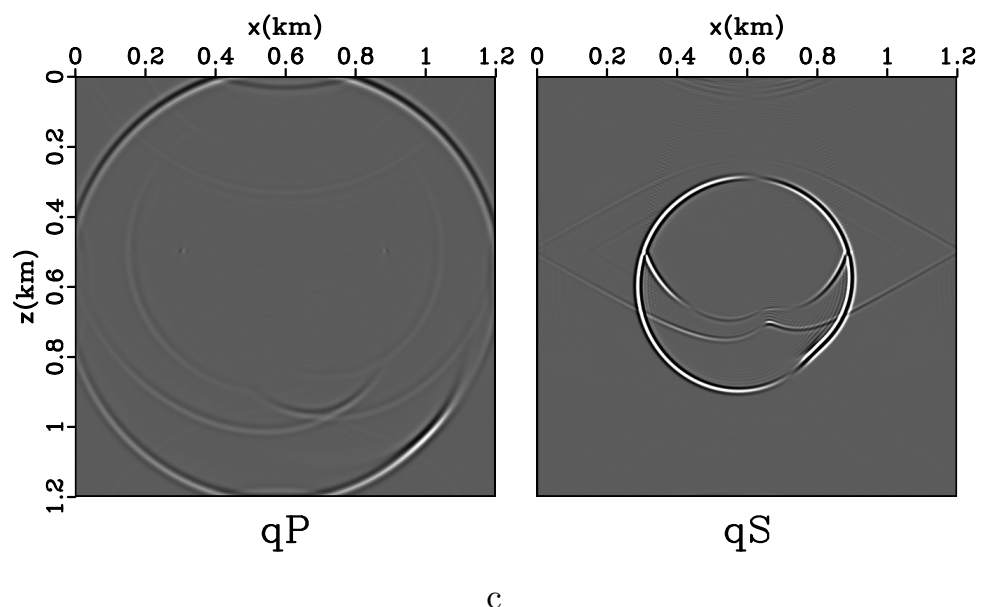

Figure 11: (a) One snapshot of the isotropic wavefield modeled with a vertical point force source at $x=0.6 \mathrm{~km}$ and $z=0.6 \mathrm{~km}$ for the model shown in Figure 10, (b) isotropic $\mathrm{P}$ and $\mathrm{S}$ wave modes separated using $\nabla \cdot$ and $\nabla \times$, and (c) isotropic $\mathrm{P}$ and $\mathrm{S}$ wave modes separated using pseudo derivative operators. Both (b) and (c) show good separation results. 


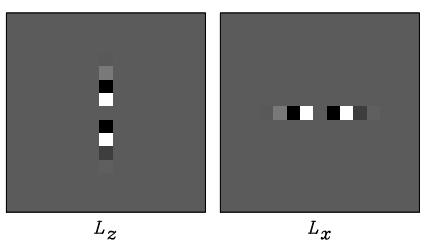

a
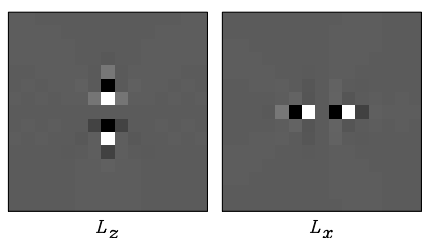

d

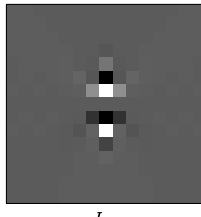

$L_{z}$

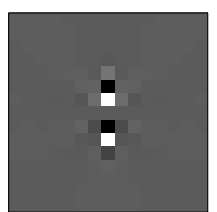

$L_{z}$

$\mathrm{b}$

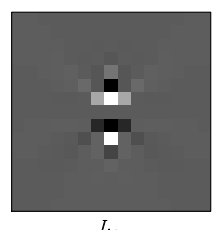

$L_{z}$

e

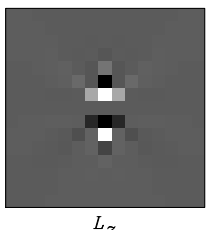

$L_{z}$
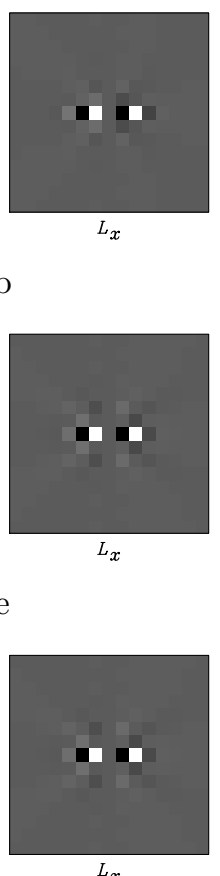

$\mathrm{h}$
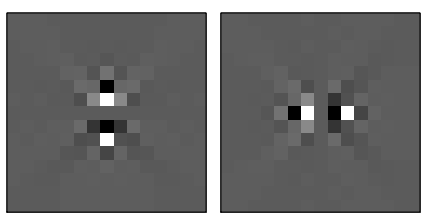

$\mathrm{C}$
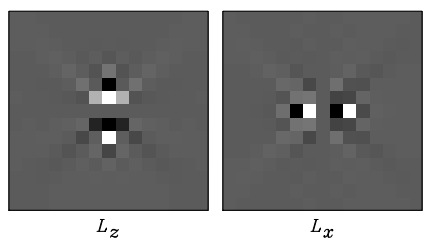

f
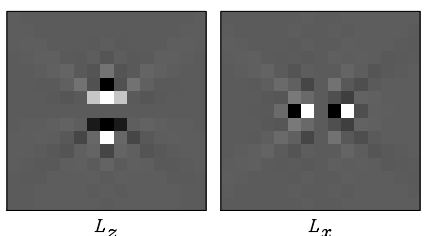

i

Figure 12: The $8^{\text {th }}$ order anisotropic pseudo derivative operators in the $z$ and $x$ directions at the intersections of $x=0.3,0.6,0.9 \mathrm{~km}$ and $z=0.3,0.6,0.9 \mathrm{~km}$ for the model shown in Figure 10. 

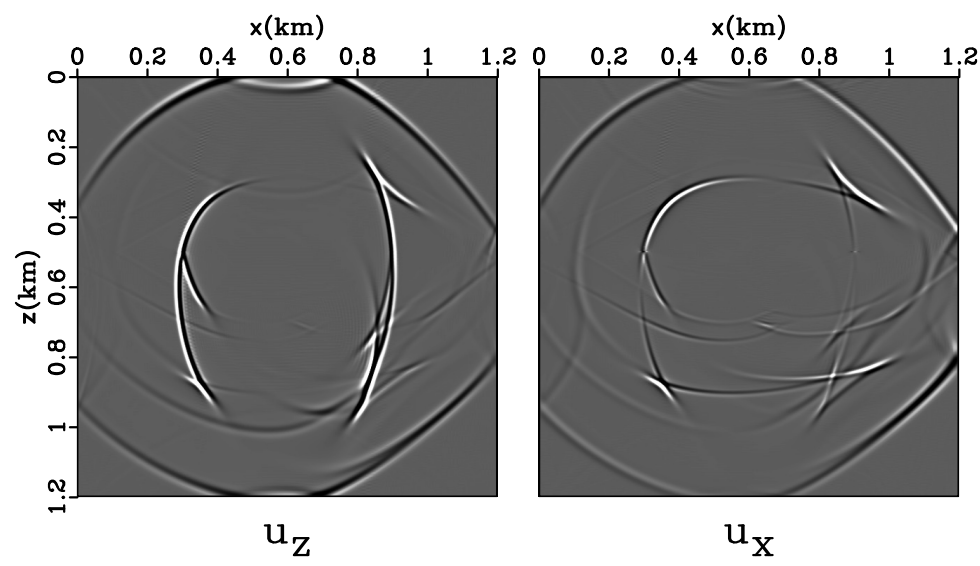

a

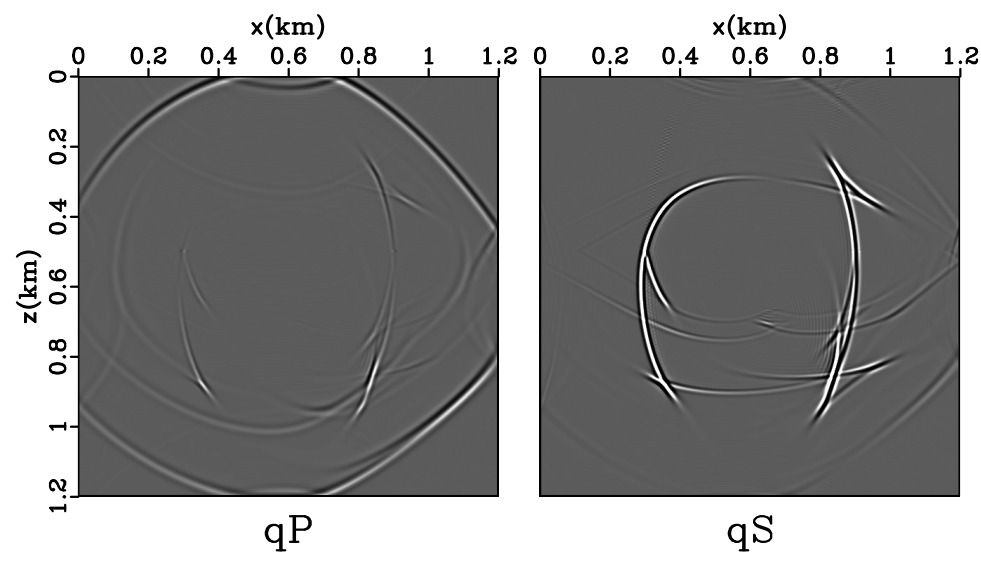

$\mathrm{b}$

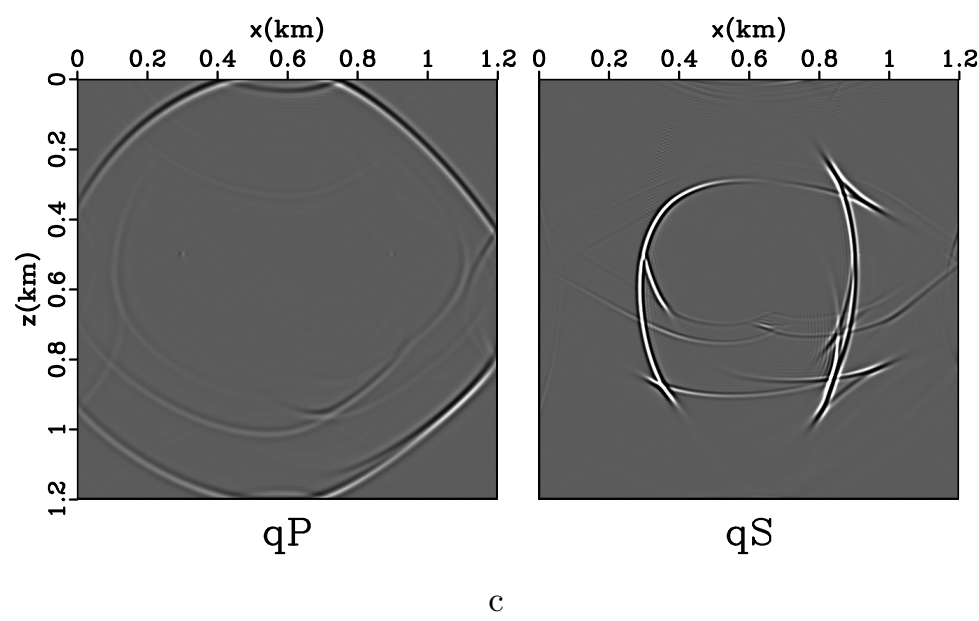

Figure 13: (a) One snapshot of the anisotropic wavefield modeled with a vertical point force source at $x=0.6 \mathrm{~km}$ and $z=0.6 \mathrm{~km}$ for the model shown in Figure 10, (b) anisotropic $q \mathrm{P}$ and $q \mathrm{~S}$ modes separated using $\nabla \cdot$ and $\nabla \times$, and (c) anisotropic $q \mathrm{P}$ and $q \mathrm{~S}$ modes separated using pseudo derivative operators. The separation of wavefields into $q \mathrm{P}$ and $q \mathrm{~S}$ modes in (b) is not complete, which is obvious at places such as at coordinates $x=0.4 \mathrm{~km} z=0.9 \mathrm{~km}$. In contrast, the separation in (c) is much better, because the correct anisotropic derivative operators are used.. 
visible at locations such as at coordinates $x=13 \mathrm{~km}$ and $z=7 \mathrm{~km}$, and $x=13 \mathrm{~km}$ and $z=4 \mathrm{~km}$ in the $q \mathrm{P}$ panel and at $x=16 \mathrm{~km}$ and $z=2.5 \mathrm{~km}$ in the $q \mathrm{~S}$ panel.

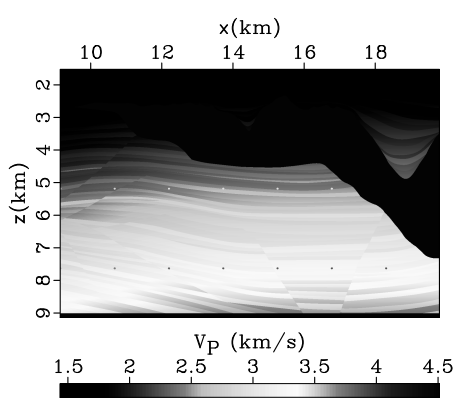

a

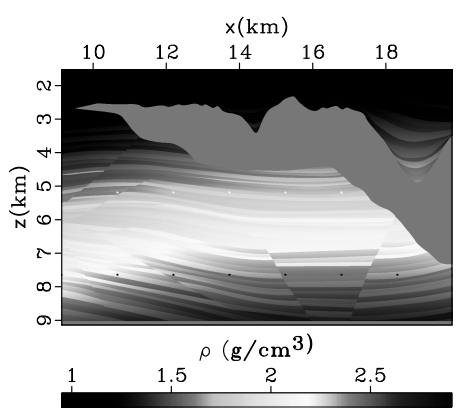

$\mathrm{C}$

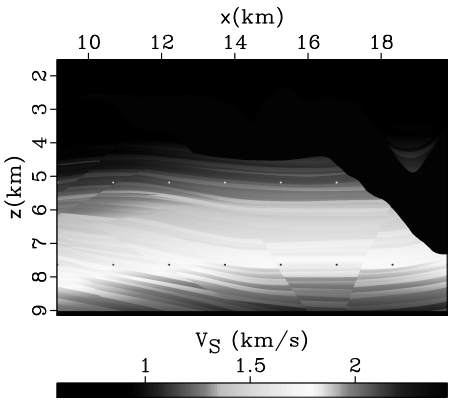

$\mathrm{b}$

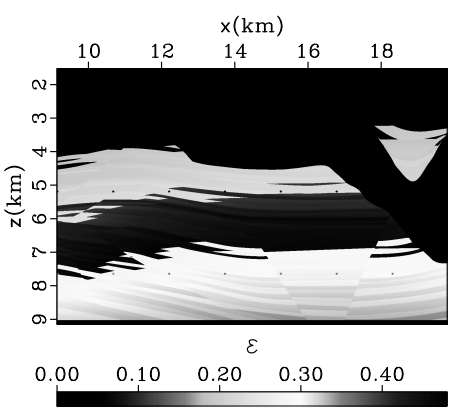

d

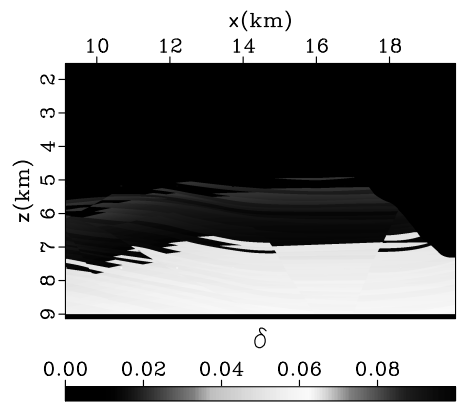

e

Figure 14: A Sigsbee 2A model in which (a) is the $\mathrm{P}$ wave velocity (taken from the original Sigsbee 2A model (Paffenholz et al., 2002) ), (b) is the S wave velocity, where $V_{P 0} / V_{S 0}$ ratio ranges from 1.5 to $2.0,(\mathrm{c})$ is the density ranging from $1.0 \mathrm{~g} / \mathrm{cm}^{3}$ to $2.2 \mathrm{~g} / \mathrm{cm}^{3},(\mathrm{~d})$ is the parameter $\epsilon$ ranging from 0.20 to 0.48 , and (e) is the parameter $\delta$ ranging from 0 to 0.10 in the rest of the model.

\section{DISCUSSION}

The separation of $\mathrm{P}$ and $\mathrm{S}$ wave-modes is based on the projection of elastic wavefields onto their respective polarization vectors. For VTI media, P and S mode polarization vectors can be conveniently obtained by solving the Christoffel equation. The 

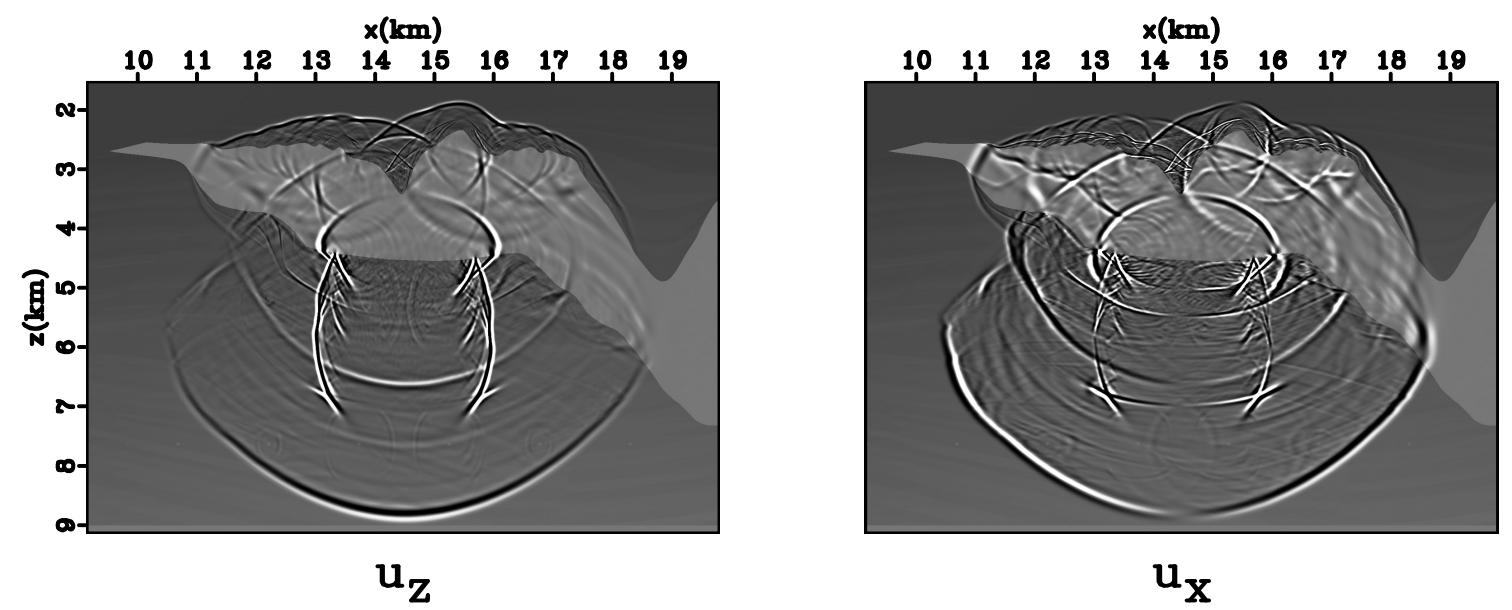

Figure 15: Anisotropic wavefield modeled with a vertical point force source at $x=$ $14.3 \mathrm{~km}$ and $z=5.3 \mathrm{~km}$ for the model shown in Figure 14.
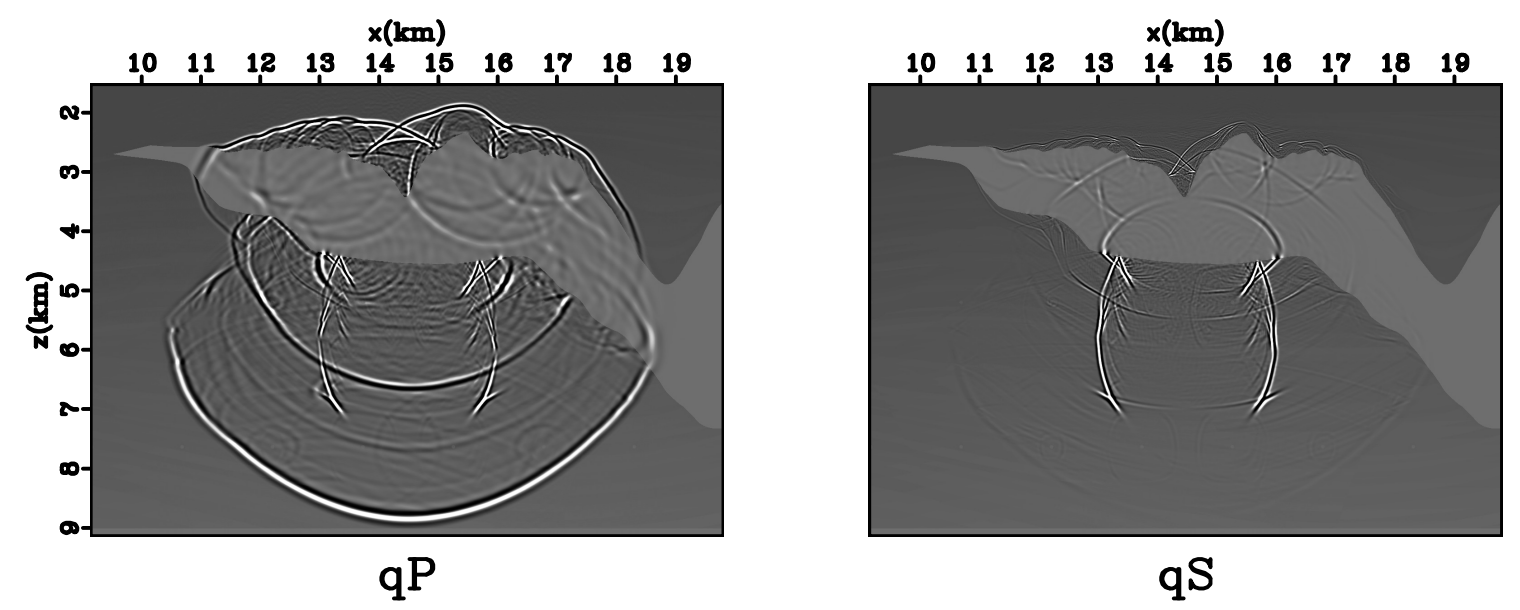

Figure 16: Anisotropic $q \mathrm{P}$ and $q \mathrm{~S}$ modes separated using $\nabla \cdot$ and $\nabla \times$ for the vertical and horizontal components of the elastic wavefields shown in Figure 15. Residuals are obvious at places such as at coordinates $x=13 \mathrm{~km}$ and $z=7 \mathrm{~km}$ in the $q \mathrm{P}$ panel and at $x=11 \mathrm{~km}$ and $z=7 \mathrm{~km}$ in the $q S$ panel. 

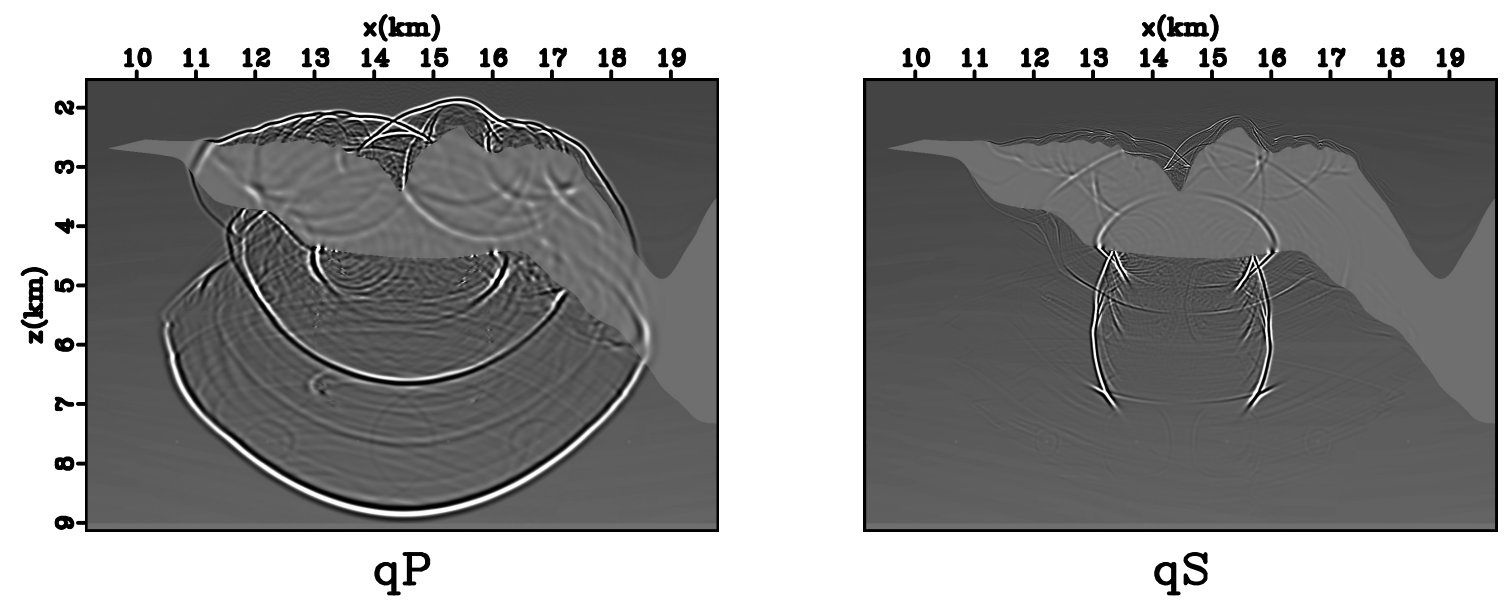

Figure 17: Anisotropic $q \mathrm{P}$ and $q \mathrm{~S}$ modes separated using pseudo derivative operators for the vertical and horizontal components of the elastic wavefields shown in Figure 15. They show better separation of $q \mathrm{P}$ and $q \mathrm{~S}$ modes.
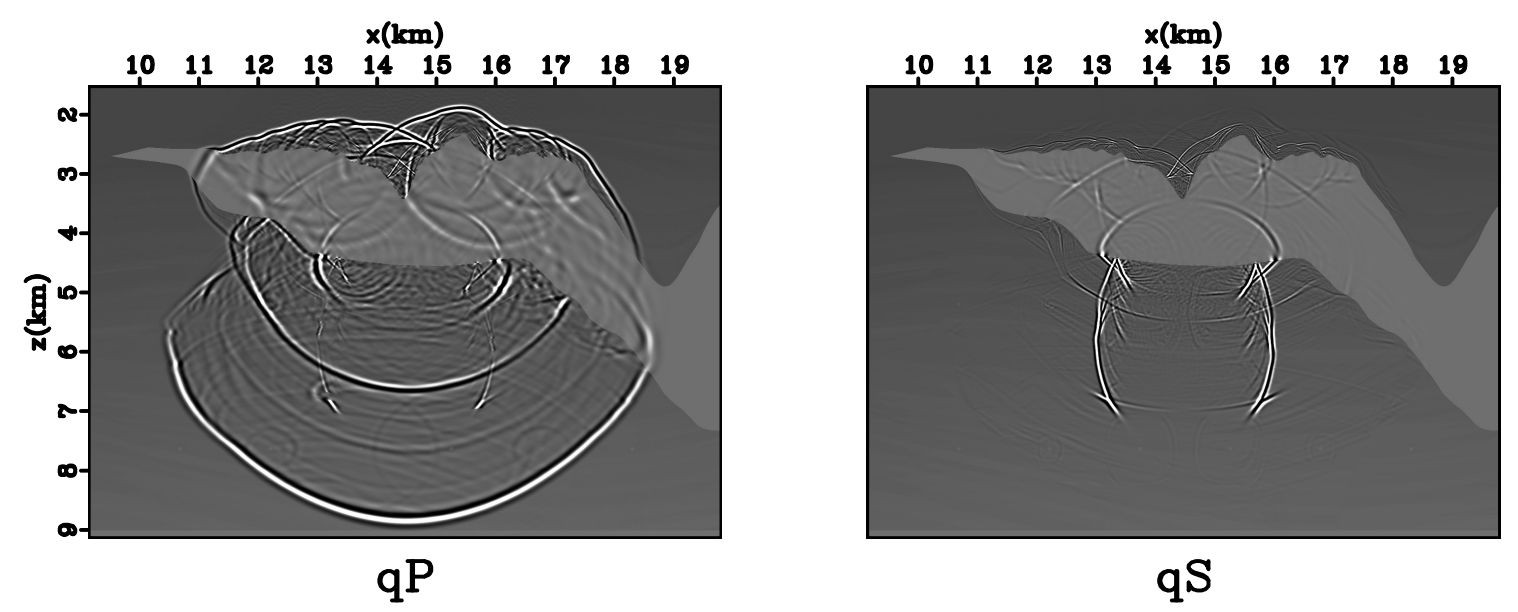

Figure 18: Anisotropic $q \mathrm{P}$ and $q \mathrm{~S}$ modes separated in the $k$ domain for the vertical and horizontal components of the elastic wavefields shown in Figure 15. The separation assumes $\epsilon=0.3$ and $\delta=0.1$ throughout the model. The separation is incomplete. Residuals are still visible at places such at coordinates $x=13 \mathrm{~km}$ and $z=7 \mathrm{~km}$, and $x=13 \mathrm{~km}$ and $z=4 \mathrm{~km}$ in the $q \mathrm{P}$ panel and at $x=16 \mathrm{~km}$ and $z=2.5 \mathrm{~km}$ in the $q \mathrm{~S}$ panel. 
Christoffel equation is a plane-wave solution to the elastic wave equation. Since the displacements, velocity and acceleration field have the same form of elastic wave equation, the separation algorithm applies to all these wavefields. The P and SV mode separation can be extended to TTI (transverse isotropy with a tilted symmetry axis) media by solving a TTI Christoffel matrix, and obtain TTI separators. Physically, the TTI media is just a rotation of VTI media.

In TI media, SV and SH waves are uncoupled most of the time, where $\mathrm{SH}$ wave is polarized out of plane. One only needs to decompose $\mathrm{P}$ and SV modes in the vertical plane. The plane wave solution is sufficient for most TI media, except for a special case where there exists a singularity point at an oblique propagation angle in the vertical plane (a line singularity in 3D), at which angle $\mathrm{SV}$ and $\mathrm{SH}$ wave velocities coincide. At this point, the SV wave polarization is not uniquely defined by Christoffel equation. S waves at the singularity are polarized in a plane orthogonal to the $\mathrm{P}$ wave polarization vector. However, this is not a problem since we define SV waves polarized in vertical planes only, therefore I remove the singularity by using the cylindrical coordinates. This situation is similar to $\mathrm{S}$ wave-mode coupling in orthorhombic media, where there is at least one singularity in a quadrant. However, as pointed out by Dellinger and Etgen (1990), the singularity in orthorhombic media is a global property of the media and cannot be removed, therefore the separation using polarization vectors in $3 \mathrm{D}$ orthorhombic media is not straightforward.

The anisotropic derivative operators depend on the anisotropic medium parameters. In Figure 19, I show how sensitive the separation is to the medium parameters. One elastic wavefield snapshot is shown in Figure $7 \mathrm{a}$ for a VTI medium with $V_{P 0} / V_{S 0}=2$ and $\epsilon=0.25, \delta=-0.29$. I try to separate the $\mathrm{P}$ and $\mathrm{SV}$ modes with (a) $\epsilon=0.4, \delta=-0.1$, (b) $\epsilon=0, \delta=-0.3$ and (c) $\epsilon=0, \delta=0$. The separation shows that parameters (a) have good separation, showing the difference in $\epsilon$ and $\delta$ is important. The worst case scenario is shown by parameters (c), where isotropy is assumed for this VTI medium.

\section{CONCLUSIONS}

I present a method of obtaining spatially-varying pseudo derivative operators with application to wave mode separation in anisotropic media. The main idea is to utilize polarization vectors constructed in the wavenumber domain using the local medium parameters and then transform these vectors back to the space domain. The main advantage of applying the pseudo derivative operators in the space domain constructed in this way is that they are suitable for heterogeneous media. The wave mode separators obtained using this method are spatially-variable filtering operators and can be used to separate wavefields in VTI media with an arbitrary degree of anisotropy. This methodology is applicable for elastic RTM in heterogeneous anisotropic media. 


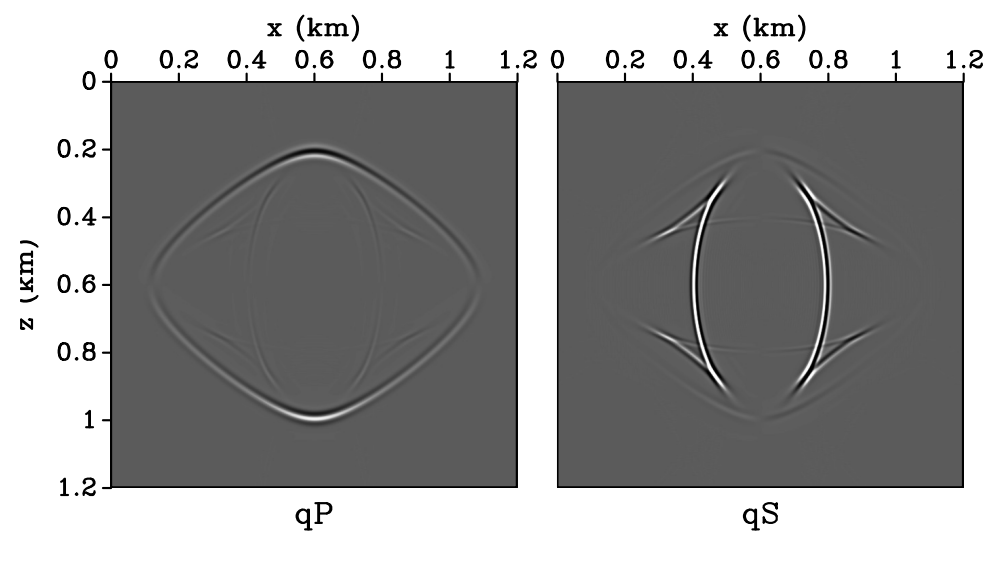

a
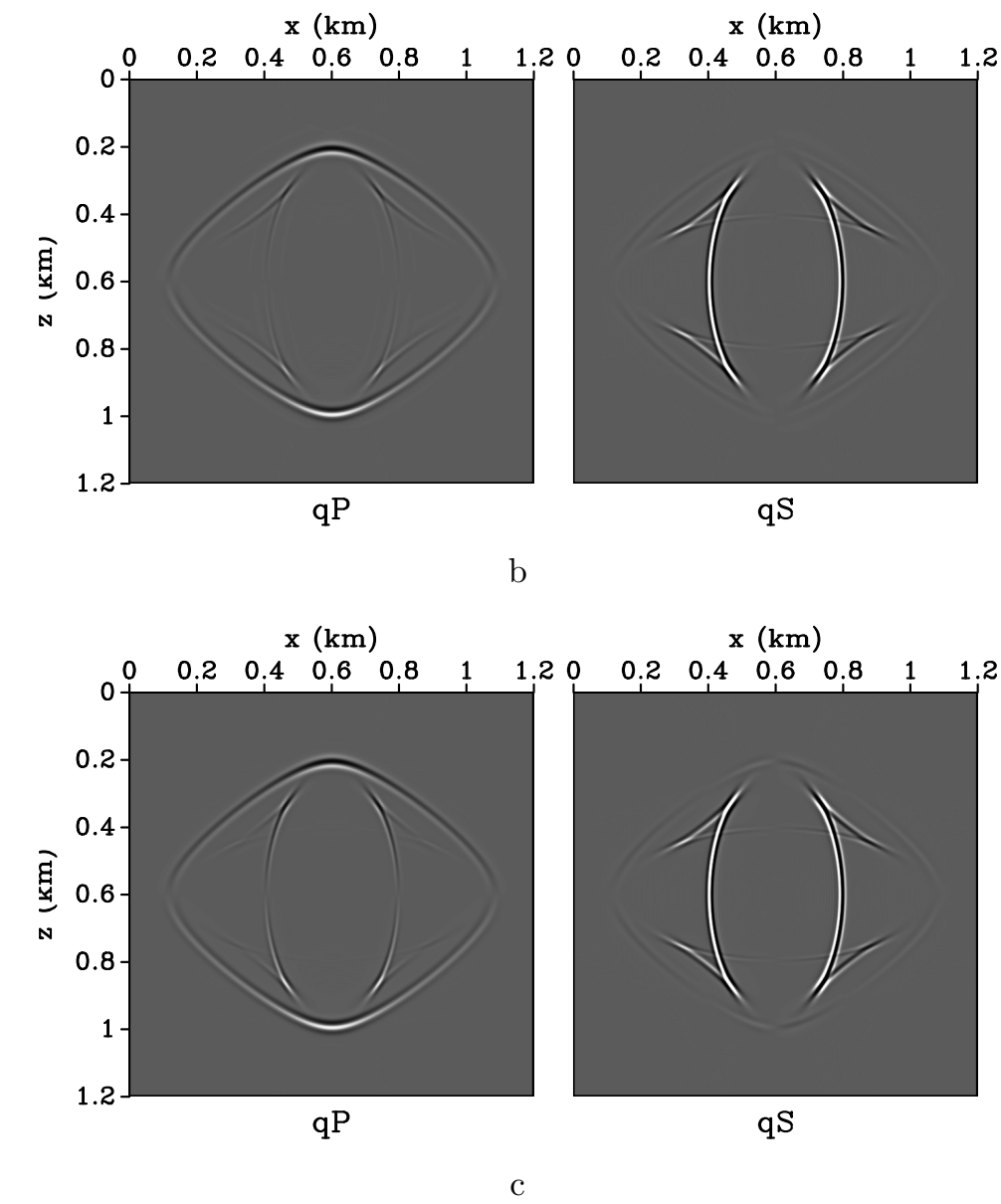

Figure 19: $\mathrm{P}$ and SV wave mode separation for a snapshot shown in Figure $7 \mathrm{a}$. The true medium parameters are $\epsilon=0.25, \delta=-0.29$. The separation assumes medium parameters of (a) $\epsilon=0.4, \delta=-0.1$, (b) $\epsilon=0, \delta=-0.3$, and (c) $\epsilon=0, \delta=0$. Hard clipping was applied to show the weak events. The plot shows that different estimate of anisotropy parameters has influence on the the wave mode separation. 


\section{ACKNOWLEDGMENT}

I acknowledge the support of the sponsors of the Center for Wave Phenomena at Colorado School of Mines. I also acknowledge reviewers Samuel Gray, Tariq Alkhalifah, and James Gaiser, whose comments and suggestions have significantly improved the manuscript. The reproducible numeric examples in this paper use the Madagascar open-source software package freely available from http://www.reproducibility. org.

\section{REFERENCES}

Aki, K., and P. Richards, 2002, Quantitative seismology (second edition): University Science Books.

Alkhalifah, T., 1998, Acoustic approximations for processing in transversely isotropic media: Geophysics, 63, 623-631.

— 2000 , An acoustic wave equation for anisotropic media: Geophysics, 65, 12391250.

Chang, W. F., and G. A. McMechan, 1986, Reverse-time migration of offset vertical seismic profiling data using the excitation-time imaging condition: Geophysics, 51, 67-84.

- 1994, 3-D elastic prestack, reverse-time depth migration: Geophysics, 59, 597-609.

Dellinger, J., and J. Etgen, 1990, Wave-field separation in two-dimensional anisotropic media (short note): Geophysics, 55, 914-919.

Etgen, J. T., 1988, Prestacked migration of P and SV-waves: SEG Technical Program Expanded Abstracts, 7, 972-975.

Fletcher, R. P., X. Du, and P. J. Fowler, 2009, Reverse time migration in tilted transversely isotropic (TTI) media: Geophysics, 74, WCA179-WCA187.

Fornberg, B., and M. Ghrist, 1999, Spatial finite difference approximations for wavetype equations: Siam Journal on Numerical Analysis.

Fowler, P. J., X. Du, and R. P. Fletcher, 2010, Coupled equations for reverse time migration in transversely isotropic media: Geophysics, 75, S11-S22.

Gray, S. H., J. Etgen, J. Dellinger, and D. Whitmore, 2001, Seismic migration problems and solutions: Geophysics, 66, 1622-1640.

Hokstad, K., 2000, Multicomponent Kirchhoff migration: Geophysics, 65, 861-873.

Paffenholz, J., B. McLain, J. Zaske, and P. Keliher, 2002, Subsalt multiple attenuation and imaging: Observations from the Sigsbee2B synthetic dataset: 72nd Annual International Meeting, SEG, Soc. of Expl. Geophys., 2122-2125.

Shan, G., 2006, Optimized implicit finite-difference migration for VTI media: SEG Technical Program Expanded Abstracts, 25, 2367-2371.

Shan, G., and B. Biondi, 2005, 3D wavefield extrapolation in laterally-varying tilted TI media: SEG Technical Program Expanded Abstracts, 24, 104-107.

Thomsen, L., 1986, Weak elastic anisotropy: Geophysics, 51, 1954-1966. (Discussion in GEO-53-04-0558-0560 with reply by author). 
Tsvankin, I., 2005, Seismic signatures and analysis of reflection data in anisotropic media: 2nd edition: Elsevier Science Publ. Co., Inc.

Yan, J., and P. Sava, 2009, Elastic wave-mode separation for VTI media: Geophysics, 74, WB19-WB32.

Zhe, J., and S. A. Greenhalgh, 1997, Prestack multicomponent migration: Geophysics, 62, 598-613. 